\title{
Graphene Growth and Characterization: Advances, Present Challenges and Prospects
}

\author{
John U. Arikpo ${ }^{1} \&$ Michael U. Onuu ${ }^{1}$ \\ ${ }^{1}$ Engineering Physics Research Group, Department of Physics/Geology/Geophysics, Alex Ekwueme Federal \\ University, Ndufu-Alike, Ebonyi State, Nigeria
}

Correspondence: Michael U. Onuu, Engineering Physics Research Group, Department of Physics/Geology/Geophysics, Alex Ekwueme Federal University, Ndufu-Alike, Ebonyi State, Nigeria. Tel: +234(0)-80-3507-2076. E-mail: michaelonuu@yahoo.com

Received: September 9, 2019

doi:10.5539/jmsr.v8n4p37
Accepted: September 26, 2019 Online Published: September 30, 2019

URL: https://doi.org/10.5539/jmsr.v8n4p37

\begin{abstract}
It is about a decade since graphene became a material for serious research by researchers in condensed matter of various nationalities making significant progress. This paper on graphene growth and characterization: advances, present challenges and prospects is therefore timely. Basic topics such as graphene and graphene technology, history and trend of graphene as well as graphene growth and synthesis have been discussed. Also presented are fundamental and mechanical properties, structural and morphological property characterization using different techniques. Graphene in biomedical and radio frequency applications, transparent electronics, integrated circuits, quantum dots, frequency multiplier, optical modulator and piezoelectricity and as a battery super capacitor are some applications and uses of graphene that have been considered. The lowering of the growth temperature of graphene has been found to be beneficial for the compartibility with other materials and processes and could also decrease the impact of cooling-induced wrinkling on the morphology of graphene; the growth on dielectric substrates; being able to resolve many problems associated with metallic growth substrates; better control of both the formation and the extension of additional layers on the graphene through substrate engineering that will result in approaches of graphene that is envisaged are some of the advances and future prospects. Also, the proposed tunable bandgap for graphene which is essential for microelectronics which contributes one of the present challenges is likely to be achieved in the very near future. Although theoretical and computational analyses have proved to have solved the zero bandgap problem of graphene, more convincing approaches that will solve the problem and give way for the fabrication of high performance graphene device are being awaited.
\end{abstract}

Keywords: Graphene Growth, Graphene Characterization, Fabrication, High Performance, Challenges and Prospects

\section{Introduction}

\subsection{Graphene and Graphene Technology}

Graphene is described in its most fundamental way as a single, thin layer of graphite, which is the soft, flaky material used in pencil lead. Graphite is an allotrope of carbon, meaning it possesses the same atoms but different in arrangement and giving the material different properties. For example, both diamond and graphite are allotrope forms of carbon, yet they have wildly different natures. Diamonds are extremely strong and ductile and can exhibit large plastic deformation, while graphite is brittle and therefore has little plasticity. From inception, the word "graphene" has the ending "-ene" which is a suffix from benzene, an aromatic hydrocarbon, signifying the chemical structure with an infinite size polycyclic hydrocarbon and consists of only one layer while the host material is the graphite (Mark et al., 2008).

Graphene could be defined as a single-atom-thick sheet of hexagonally or honeycomb lattice arrayed $\mathrm{sp}^{2}$ bonded carbon atoms Figure 1 and the building block of other allotropes of carbon that got a significant attention due to its unique electronic (Schedin et al., 2007), mechanical (Lee et al., 2008a), and thermal (Xiao et al., 2011) properties all derived from the unique details of its electronic band structure. Eda et al. (2008) \& Wu et al. (2010) reported that due to its flexibility, graphene provides infinite possibilities in various fields and the peculiar dispersion relation of carbon's $\pi$ electrons is the reason for its unique properties. Furthermore, graphene material has been proved to be the most stretchable crystal, the most impermeable material, even to helium; it has a record thermal 
conductivity 10 times as high as copper, and the highest intrinsic electron mobility, about 100 times that of silicon (Geim \& Neovoselov, 2007).

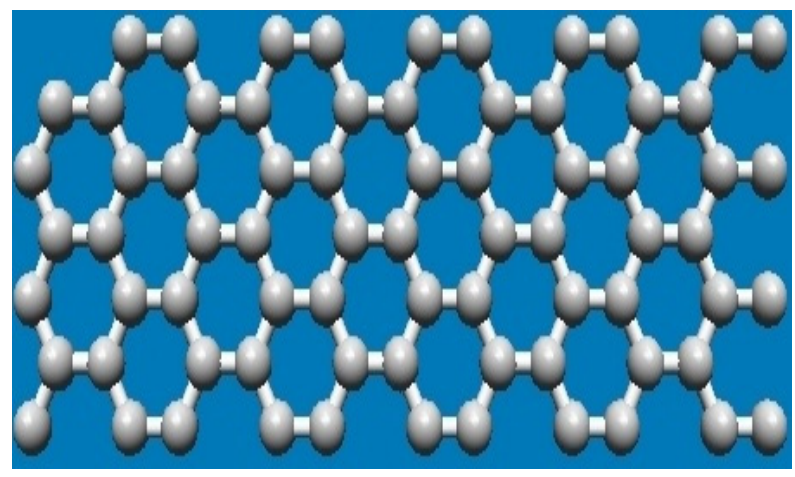

Figure 1. A diagram of the honeycomb lattice structure of graphene (Nanotube modeler@2005-2010, JCrystalsoft)

According to Wallace (1947), the theoretical study of graphene is believed to have started in 1940s as a text book meant for calculations in solid state physics. The study predicted the electronic structure and noted the linear dispersion relation in graphene sheet. The contribution of McClure in 1956 also proved that graphene (or " $2 \mathrm{D}$ graphite") studies have been in existence for sixty years and widely used for describing properties of various carbon-based materials. McClure (1956) proposed the wave equation for excitations and the similarity to the Dirac equation which was discussed in Semenoff (1984) and DiVincenzo \& Mele (1984) while Boehm et al. (1962) synthesized the first isolated carbon sheets. Their work employed thermal reduction of graphene oxide in solution to prepare one atom thick layers of carbon, but the result from their investigation did not attract any significant attention at the time. It was not until 40 years later that graphene became the focus of interest due to its attractive physical properties and the potential applications these properties offer for the future. Although scientists knew that one atom thick, two-dimensional crystal graphene existed, but no-one had worked out how to extract it from graphite, instead it took 60 years as earlier discussed to experimentally obtain a few sheets of micrometer-sized high-quality flakes weighing picograms (Novoselov et al., 2004) and this was discovered in 2004 by two researchers at the University of Manchester, Prof Andre Geim and Prof Kostya Novoselov who first used adhesive tape to isolate graphene from graphite through mechanical exfoliation method. They published their results in October of 2004 in Science journal (Novoselov et al., 2004). In this paper they described the fabrication, identification and Atomic Force Microscopy (AFM) characterization of graphene.

Mechanical exfoliation method was used for extracting thin layers of graphite from a graphite crystal with Scotch tape and then transferred these layers to a silicon substrate. This method was first suggested and tried by Ruoff's group (Lu et al., 1999) who were, however, not able to identify any monolayers. The Manchester group succeeded by using an optical method with which they were able to identify fragments made up of only a few layers. Today graphene sheets are being produced in hundreds of tones and tens of thousands of square meters and this is recorded as a serious breakthrough in the world of graphene research during the $21^{\text {st }}$ century.

Graphene technology demonstrates the latest graphene research and development on practical commercial applications of tools and methods in the material's growth. It also focuses on the commercial and practical applications of this material and its different forms, such as graphene sheets, nanoplatelets, graphene nanotubes, dispersions and composites. This Technology has so far given vital information on commercial and practical applications of graphene and graphene related materials. The contents include full research papers, short communications, case studies and comprehensive review articles. Advancements in graphene research increase on a daily basis as new materials are discovered in piecemeal. Many outstanding contributions have been made from great minds from then till date with emergence of graphene materials having promising features aimed at changing the world.

\subsection{History and Trend of Graphene}

Graphene was first produced by mechanical exfoliation of graphite as previously discussed. This method provided a small amount of high quality samples for fundamental studies. Shortly after that, several methods were involved in the synthesis of graphene films which are grouped into bottom-up and top-down approaches with carbon-containing molecules and graphite as initial materials, respectively. The choice of a method for the 
synthesis of graphene is narrowed down to its cost, amount, size of sheets, quality of sheets, chemical modification and compatibility with commercial chip fabrication process. The technique developed by Andre Geim and Konstantin Novoselov and their related research on graphene, awarded them a Nobel Prize in Physics in 2010. This relatively simple preparation method led to a large increase of interest, since research groups all over the world were now able to produce and investigate graphene samples. This high interest is also reflected by the number of scientific publications related to graphene research, as shown in Figure 2. Advancements in graphene research increased exponentially from 2004 to 2014, the number of publications with titles containing 'graphene' per year increases with the years and the trend of this increment is stimulated by the extraordinary properties of graphene. New methods to produce the material were also developed in the hope of enabling graphene to play an important role in future every-day applications.

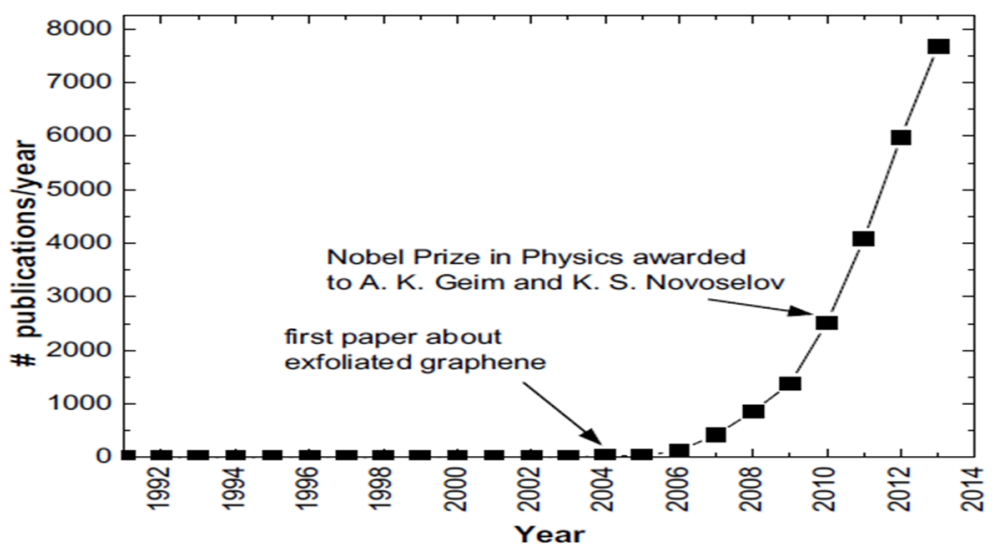

Figure 2. Number of publications (article, proceeding paper, review or letter) with the title containing 'graphene' per year (Thomas Reuters, 2013)

It is said that if the 20th century was the age of plastics, the $21^{\text {st }}$ century seems set to become the age of graphene. It is the lightest, thinnest, best heat and electricity conducting material ever discovered.

It promises to revolutionize everything from computing to car tyres and solar cells to smoke detectors. Making solar cell 50 to 100 times more efficient, being 50 to 100 times faster than semiconductors, producing aircraft that is $70 \%$ lighter, graphene is a battery that charges 10 times faster and stores 10 times more power.

Also, being 200 times stronger than steel, thinner than a sheet of paper and more conductive than copper, companies such as Samsung, Nokia and IBM are already developing graphene-based replacements for touch screens, transistors, and flash memories though the work is at a very early stage.

\subsection{Graphene Growth and Synthesis}

Despite the advances in graphene research, obtaining a controlled method on film size that can produce larger crystalline graphene domain is still not available. This demands that researches on graphene growth still need to be improved. Graphene production could be in small sheets or large-area films. Small graphene sheets can be used in composites, functional coatings, conductive inks, batteries and supercapacitors (Zhu et al., 2010; Novoselov et al., 2012). Large-area graphene films can be used as transparent electrodes in touch panels, displays and photovoltaic devices with potentially low cost, and more importantly, they are expected to be used in next-generation electronics and optoelectronics such as flexible and wearable devices (Bonaccorso et al., 2010; Novoselov et al., 2012). There are a number of methods used for the preparation of graphene, these methods are applied with regards to quality and size of the flakes or controllability of the resulting coating and where the synthesized material can find best application.

\subsection{Synthesis of Graphene}

Synthesis of graphene refers to any process for fabricating graphene, depending on the desired size, purity and crystallinity of the final product. Inspite of the numerous achievements in graphene research, the quality of the sample and large scale production for industrial and commercial purposes still suffer for lack of appropriate synthesis method in the control of the film size. The investigation of structural evolution and growth mechanism can also provide guidance to produce the desired graphene (Jafari et al., 2015). Many parameters in graphene growth have been investigated. However, high quality single layer and multilayer graphene can be synthesized by 
tuning the growth conditions of different gaseous, liquid or solid carbon sources (Kraus et al., 2013), different dilution gases (Kraus et al., 2013), (Zhang et al., 2012), with different flow rates (Kumar et al., 2012), different temperature, process pressure (Zhang et al., 2012), cooling rates (Sarno et al., 2013), as well as catalysts (Kumar et al., 2012), catalyst's thickness, crystallographic orientation (Sarno et al., 2013), and purity states (Sarno \& Wu et al., 2013).

\section{Synthesis Techniques/Methods}

There are basically seven methods of synthesizing graphene, they include:

\subsection{Mechanical Exfoliation}

This is the first and oldest method used to obtain single layer graphene. In this method, individual graphene sheets are separated from graphite. In 2004, two scientists namely Andre Geim and Konstantin Novoselov of University of Manchester used Scotch Tape and stuck it on graphite and which result in the peeling off of many layers. Next, they used a second piece of tape to peel a few graphite layers off of the first piece of tape. The process continued for some time until the last piece of tape was stuck to a flat silicon wafer which was later peeled off. The layers remaining on the wafer were found to be a single atom thick graphene. The method is simple and not expensive when compared with other techniques.

Mechanical exfoliation has the constraint of control in the uniformity of the layers especially when the requirement is continuity in layers. The graphene sample produced from this method is irregular in shape and has low commercial value as well as limited application in electronic devices.

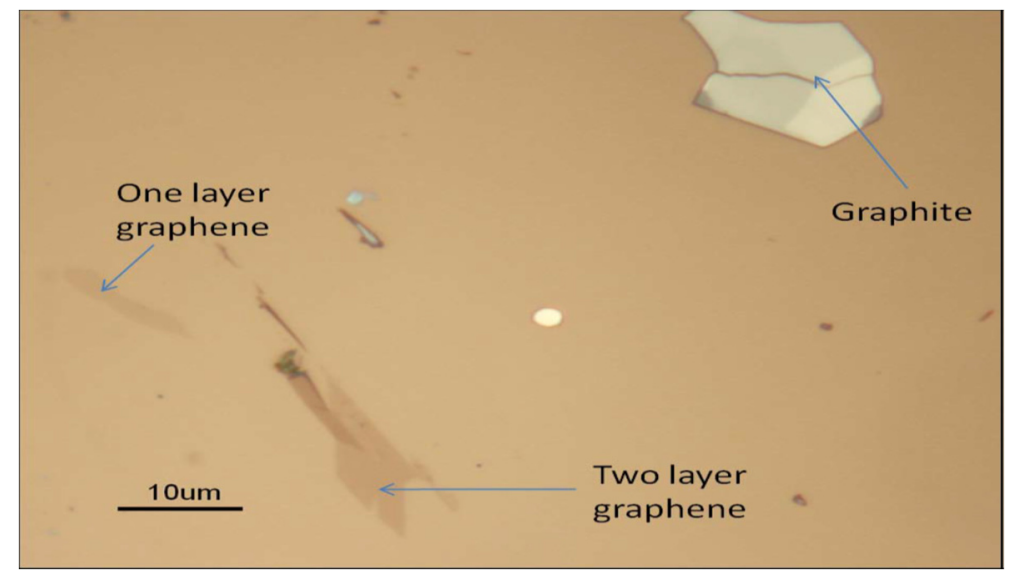

Figure 3. Mechanically exfoliated graphene on $\mathrm{SiO}_{2}$ imaged with white light using an optical microscope (Jones \& Safron, 2004)

\subsection{Epitaxial Growth on Silicon Carbide}

In this method, silicon carbide $(\mathrm{SiC})(0001)$ is reduced to graphene by annealing at a very high temperature $\left(1000-1600^{\circ} \mathrm{C}\right)$ and vacuum. Epitaxial graphene on insulating substrate has been demonstrated to exhibit high mobilities, especially multilayered films. With this method, the control of graphene layer becomes simple but difficult to obtain large area films (Sutter, 2009). Recently, single layered SiC converted graphene over a large area has been reported and shown to exhibit outstanding electrical properties (Wu et al., 2009). Graphene grown on the following insulating substrates such as: plastic foils, glass or $\mathrm{SiO}_{2} / \mathrm{Si}$ wafers are better used for electronic applications. According to Kim et al. (2009), Bae et al. (2010) \& Li et al. (2009) different methods are used in the transfer of as-grown graphene from metallic surfaces onto desired insulating substrates.

\subsection{Chemical Vapour Deposition}

Chemical vapour deposition (CVD) involved the decomposition of fluid (gas and liquid sprays) at high temperature to form either thin films on substrates or powders through filters (Atta et al., 2015).

It is also a chemical process used to produce high quality and high performance solid materials. The process is often used in the semiconductor industry to produce thin films. The mechanism behind the growth of graphene using chemical vapour deposition (CVD) is deposition of vapour species from various chemical reactions. The chemical reactions play a vital role and acts as one of the distinctive features of this process. CVD growths have largely proved to be a successful means for growing uniform and large area graphene. This method is used when 
the need for large-scale production and high quality graphene samples arises. The disadvantage with this method is in the area of carrier transfer onto a different substrate which eventually leads to folding, defect, tearing and wrinkles in the graphene sample, otherwise the method is a straightforward process and considered to be the most reliable for large scale production for commercial and industrial use, respectively. There are many forms of CVD including: hot wire CVD, thermal CVD, plasma enhanced (PE) CVD (An et al., 2011), radio-frequency (RF) CVD, ultrasonic spray pyrolysis (USP) among many derivatives (Terasawa \& Saiki, 2012).

\subsection{Arc Discharge Method}

Subrahmanyam et al. (2009) \& Rao et al. (2010) reported on the synthesis of graphene by Arc discharge method with the evaporation of graphite in the presence of hydrogen. Their method of synthesis is less expensive and has the ability to produce sheets with two to three layers having flake size of $100-200 \mathrm{~nm}$. Just after that, Rao \& Sood (2013) demonstrated that the presence of $\mathrm{H}_{2}$ during arc discharge process terminates the dangling carbon bonds with hydrogen and prevents the formation of closed structures. This method of synthesis involved the use of high current (above 100A), high voltage ( $>50 \mathrm{~V})$, and high pressure of hydrogen (above 200Torr) needed for obtaining graphene in the inner walls.

\subsection{Reduction of Graphite Oxide}

Chemical reduction of graphite oxide is one of the established procedures to prepare graphene in large quantities (Hummers \& Offeman, 1958). Graphite oxide when ultrasonicated in water forms a homogeneous colloidal dispersion of predominantly single layer graphene oxide in water. Dreyer et al. (2010) were able to show how reduced graphene oxide with properties similar to that of graphene could be prepared through chemical, thermal or electrochemical reduction pathways. Meanwhile most strong reductants have slight to strong reactivity with water, hydrazine monohydrate does not, making it an attractive option for reducing aqueous dispersions of graphene oxide (Stankovich et al., 2007). Synthesis addition of $\mathrm{H}_{2}$ occurs across the alkenes, coupled with the extrusion of nitrogen gas. Large excess of $\mathrm{NaBH}_{4}$ has also been used as a reducing agent (Shin et al., 1987). Other reducing agents used include phenyl hydrazine (Pham et al., 2010), hydroxylamine (Zhou et al., 2011), glucose (Zhu et al., 2010), ascorbic acid (Zhang et al., 2010), hydroquinone (Wang et al., 2008), alkaline solutions (Fan et al., 2008) and pyrrole (Amarnath et al., 2011).

Graphene can also be synthesized in large scale using electrochemical reduction means (Guo et al., 2009; Sundaram et al., 2011). Their investigation shows that reduction is initiated at $-0.8 \mathrm{~V}$ and is completed at $-1.5 \mathrm{~V}$, with the formation of black precipitate onto the bare graphite electrode. Zhou et al. (2009) coupled electrochemical reduction with a spray coating technique to prepare large-area and patterned RGO films with thicknesses ranging from a single monolayer to several micrometers on various conductive and insulating substrates.

\section{Graphene Transfer}

Transfer of graphene unto a substrate forms an integral part of the growth process as it plays a significant role in the choice of temperature and the quality of graphene produced. The film grown through CVD is very important since it promotes conductivity thereby enhancing the free flow of electrons in the entire film. In order for electrical current to flow through graphene devices as opposed to being shorted out by a conducting substrate, graphene should be removed from the conducting catalyst surface of $\mathrm{Cu}, \mathrm{Ni}, \mathrm{Rb}$, etc. and transferred onto an insulating surface (Kedzierski et al., 2009). Not all the transfer techniques are applicable for CVD graphene. Graphene transfer techniques suitable for CVD graphene are usually done by etching the underlying catalyst and then scooping the graphene directly or using some kind of media (thermoplastic materials) such as Polymethyl methacrylate (PMMA), Polydimethylsiloxane (PDMS), Polyethylene terepthalate (PET), dielectric material and thermal tape. Not all techniques are appropriate for used such as mechanical exfoliation or chemical exfoliation when dealing with multi-layer graphite as precursor material.

The Transfer of graphene to $300 \mathrm{~nm} \mathrm{SiO} / 2 \mathrm{Si}$ wafer substrates was carried out as reported by $\mathrm{Li}$ et al. (2009) and the following investigations were made, a layer of poly (methyl methacrylate) (PMMA) (MicroChem 495 PMMA A6) was spin-cast on $\mathrm{Cu}$ foils after graphene growth at $3000 \mathrm{rpm}$ for 30 second and baked at $180 \mathrm{uC}$ for $2 \mathrm{~min}$. Secondly, oxygen plasma was used to remove graphene on the back side of $\mathrm{Cu}$ foils. Next, copper was wet-etched in an aqueous solution of $\left(\mathrm{NH}_{4}\right)_{2} \mathrm{~S}_{2} \mathrm{O}_{8}$ for about 8hours, resulting in graphene/PMMA films floating in the etchant. Then, the films were rinsed in ultrapure water for three times, transferred to $300 \mathrm{~nm} \mathrm{SiO}_{2} / \mathrm{Si}$ substrates and baked at $50 \mathrm{uC}$ for half an hour. Finally, PMMA was removed by annealing the samples at $420 \mathrm{uC}$ for 180 mins under hydrogen $(600 \mathrm{sccm})$ and argon $(300 \mathrm{sccm})$ gas flow. 


\subsection{Production}

According to Feynman (1959), graphene was first produced by mechanical exfoliation as previously discussed followed by other methods which were employed for the synthesis of graphene sheet. These other techniques can be categorized into bottom-up and top-down methods with carbon containing molecules and graphite as the fundamental material. The method of synthesis is dependent on the cost, size and quality of sheets, chemical modification, and compatibility with commercial chip fabrication. Table 1 shows a summary of some of the innovative efforts put in place to synthesize a quality graphene. They include: Liquid suspension graphene oxide followed by chemical reduction (Castro-Neto et al., 2009), liquid-phase exfoliation (Stankovich et al., 2007), epitaxial growth by thermal desorption of $\mathrm{Si}$ atoms from the $\mathrm{SiC}$ surface (Hernandez et al., 2008), epitaxial growth by chemical vapour deposition on transition metals (Emtsec et al., 2009), (Sutter, 2008), solvothermal synthesis (Kim et al., 2009), and unzipping carbon nanotubes (Choucair et al., 2009) are among the reported methods.

Table 1. Comparisons of different methods for graphene production (Taghioskoui, 2009)

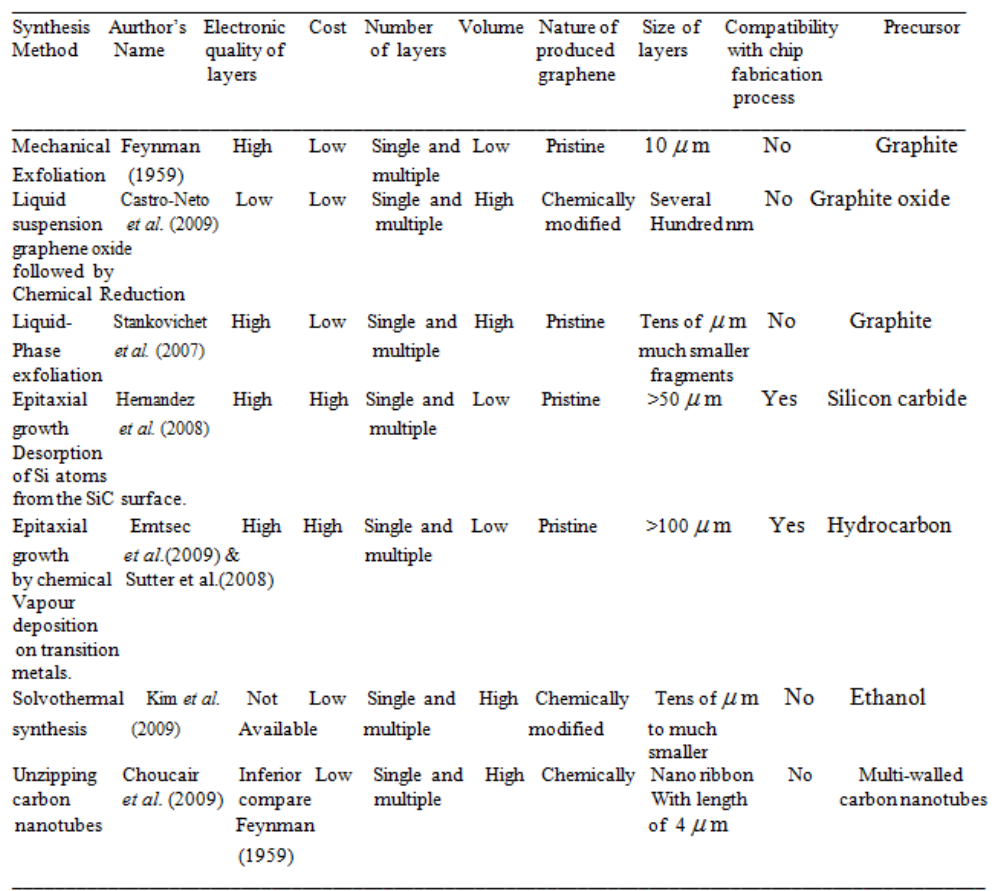

\section{Properties of Graphene}

The fundamental properties of graphene being a two-dimensional, one atom thick crystal built of sp $^{2}$ hybridized carbon attracted the attention of many researchers according to (Charlier et al., 1992). Its unit cell consists of two carbon atoms, arranged in a hexagonal honeycomb lattice Figure 4(a).

The two atoms in the unit cell form the equivalent sublattices $\mathbf{A}$ and $\mathbf{B}$. The two lattice vectors $\mathrm{a}_{1}$ and $\mathrm{a}_{2}$ can be written as (Castro-Neto et al., 2009):

$$
a_{1}=\frac{a_{C-C}}{2}\left(\frac{3}{\sqrt{3}}\right), \quad a_{2}=\frac{a_{C-C}}{2}\left(\frac{3}{-\sqrt{3}}\right)
$$

where $a_{C-C}$ represents the carbon-carbon bond distance and is about $1.42 \AA$ and the reciprocal-lattice vectors are given by (Castro-Neto et al., 2009).

$$
b_{1}=\frac{2 \pi}{3 a_{C-C}}\left(\frac{1}{\sqrt{3}}\right), \quad b_{2}=\frac{2 \pi}{3 a_{C-C}}\left(\frac{1}{-\sqrt{3}}\right)
$$


Reich et al. (2002) and Hattab et al. (2012) proposed a theoretical calculation to obtain a value for the length of the lattice vectors (and therefore the graphene's lattice constant) of about $2.461 \AA$ and this constant value is the same as bulk graphite. The positions of the three nearest neighbours of each carbon atom are given by the vectors (Castro-Neto et al., 2009):

$$
\delta_{1}=\frac{a_{C-C}}{2}\left(\frac{1}{\sqrt{3}}\right), \quad \delta_{2}=\frac{a_{C-C}}{2}\left(\frac{1}{-\sqrt{3}}\right), \quad \delta_{3}=-a_{C-C}\left(\frac{1}{0}\right)
$$

where $\delta_{1}, \delta_{2}$ and $\delta_{3}$ are the unit vectors.

Graphene sheet has carbon atoms arranged in $\mathrm{sp}^{2}$ hybridization with three in-plane, one from the s-orbitals and the out-of-plane from the $p$-orbitals shown in Figure 4(b). In this way, each of the carbon atoms form an equivalent s-bond to each of its three neighboring atoms. The bonding energy of one C-C bond in graphene amounts to $4.93 \mathrm{eV}$ (Brenner et al., 2002).

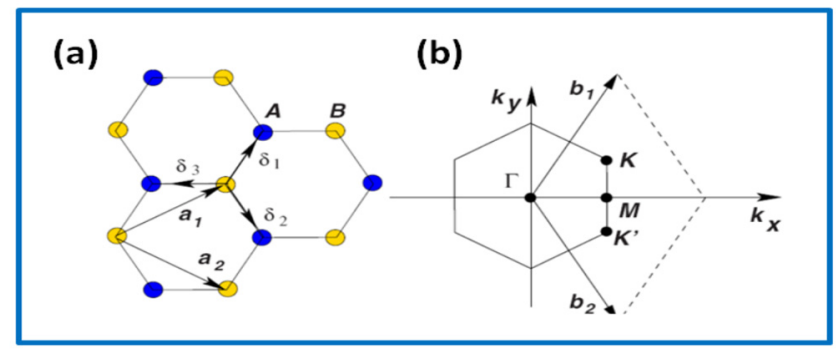

Figure 4. (a) Honeycomb lattice structure of graphene at the left made up of two interpenetrating triangle lattices where $\mathrm{a}_{1}$ and $\mathrm{a}_{2}$ are the lattice unit vectors and $\delta_{i}, i=1,2,3$ are the nearest-neighbour vectors. (b) At the right is its Brillouin zone, the Dirac cones are located at the $\mathrm{K}$ and $\mathrm{K}^{\mathrm{I}}$ points. Atoms in the two different sublattices $\mathrm{A}$ and

$\mathrm{B}$ are depicted in blue and yellow, respectively (Castro- Neto et al., 2009)

The two points $K$ and $K^{I}$ at the corners of the graphene Brillouin zone $\mathrm{B}_{\mathrm{Z}}$ are also called Dirac points in solid state physics. Their positions in momentum space are given by (Castro- Neto et al., 2009).

$$
K=\left(\frac{2 \pi}{3 a_{C-C}}, \frac{2 \pi}{3 \sqrt{3 a_{C-C}}}\right), \quad K^{I}=\left(\frac{2 \pi}{3 a_{C-C}}-\frac{2 \pi}{3 \sqrt{3} a_{C-C}}\right) .
$$

The three nearest neighbour vectors in real space are given by

$$
\delta_{1}=\frac{a_{C-C}}{2}(1, \sqrt{3}), \delta_{2}=\frac{a_{C-C}}{2}(1,-\sqrt{3}), \delta_{3}=-a_{C-C}(1,0)
$$

while the six second neighbours are located at

$$
\delta_{1}^{I}= \pm a_{1 C-C}, \delta_{2}^{I}= \pm a_{2 C-C}, \delta_{3}^{I}= \pm\left(a_{2 C-C}-a_{1 C-C}\right)
$$

From Figure 4, we can define the unit vector, and the reciprocal lattice vectors. This calculation leads to the following energy dispersion in graphene (Bonaccorso et al., 2010).

$$
E_{g 2 D}(\vec{k})= \pm t \sqrt{1+4 \cos \frac{\sqrt{3 k_{x} a_{C-C}}}{2} \cos \frac{k_{y} a_{C-C}}{2} 4 \cos ^{2} \frac{k_{y} a_{C=C}}{2}}
$$

In this case, the energies have the values of $\pm 3 t, \pm t$ and 0 , respectively at the high symmetry points, $\Gamma, M$ and $K$ in the Brillouin zone. The positive part of the energy dispersion describes the $\pi^{*}$ anti-bonding energy band and the negative part is the $\pi$ bonding energy band. Interestingly, the $\pi^{*}$ anti-bonding and $\pi$ bonding bands are degenerate at the $K$ points through which the Fermi energy passes. The first significant feature of this 
result is that, since the energy band is exactly symmetric about the point $E=E_{2 p}=0$, and this condition is met only at Dirac point. It follows that for exactly half filling of the band the density of states at the Fermi level is exactly zero and undoped graphene is a perfect semimetal. At zero doping, the lower half of the band is filled exactly up to the Dirac Point. If by applying a suitable "gate" voltage to the graphene relative to the substrate we induce a nonzero charge, this is equivalent to injecting a number of electrons in the upper half or holes in the lower half of the Dirac cones.

Figure 5 shows energy dispersion of graphene on momentum space. In particular, $K$ points where valence band and conduction band are connected called "Dirac points". This property forms one of the unique features of graphene. Near the $K$ and $K^{I}$ points, the energy dispersion has a circular cone shape which, to a first order approximation, is given by (Cooper et al., 2012).

$$
\begin{gathered}
E(\vec{k})= \pm \hbar v_{F}|k| \\
v_{F}=\frac{3 a t}{2 \hbar} \approx 10^{6} \mathrm{~ms}^{-1}
\end{gathered}
$$

where $v_{F}$ is the Fermi velocity, $m$ is the electron mass, $\hbar$ is the reduced planks constant, at is the energy value at symmetry points, $\mathrm{a}$ is the lattice distance. It is observed that the wave vector $\mathrm{k}$ is measured from the $\mathrm{K}$ and $\mathrm{K}$ ' points. This kind of energy dispersion is different from that of a conventional 2D system, due to the fact than energy dispersion is not related to mass.

$$
E(\vec{k})=\frac{\hbar^{2} k^{2}}{2 m}
$$

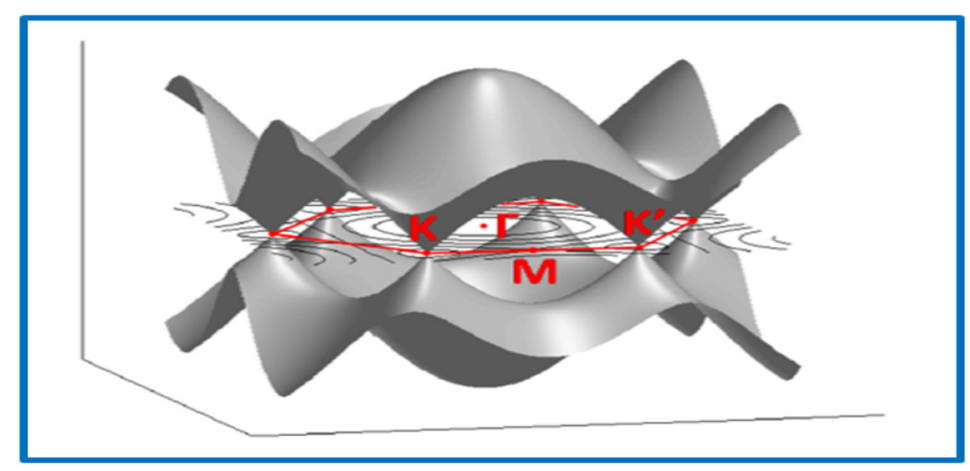

Figure 5. First Brillouin zone and band structure of graphene (Cooper et al., 2012)

The vertical axis represents the energy coordinate, while the horizontal axis is momentum space on the graphene reciprocal lattice. The first Brillouin zone of graphene is illustrated in the horizontal plane and labeled with some points of interest. Especially, $\mathrm{K}$ and $\mathrm{K}$ ' are known as the Dirac points, which connect the valence band and the conduction band (Cooper et al., 2012).

\subsection{Mechanical Properties}

Lee et al. (2008b) used nano-indentation technique in an atomic force microscope to measure the mechanical properties of graphene. They found the Young's modulus of graphene to be $1.0 \pm 0.1 \mathrm{TPa}$ on a film with thickness $0.335 \mathrm{~nm}$ and mechanical strength of more than $100 \mathrm{GPa}$ precisely $\sigma_{\text {int }}=130 \mathrm{GPa}$ and third-order elastic stiffness of $\mathrm{D}=-2.0 \mathrm{TPa}$ with a breaking strength of $\sim 40 \mathrm{~N} / \mathrm{m}$, and second-and third-order elastic stiffnesses of $340 \mathrm{Nm}^{-1}$ and $690 \mathrm{Nm}^{-1}$, respectively. Further investigation shows the elastic modulus of monolayer graphene sheet performed via chemical reduction in graphene oxide to be $0.25 \mathrm{TPa}$ by indentation of an atomic force microscopy tip at the centre of a suspended graphene sheet. Investigations from previous research proved graphene to be the strongest material ever studied, probably because of its robust lattice of $\mathrm{sp}^{2}$ bonding. The sigma $(\delta)$ bonds as seen previously in Figure 4 are responsible for the extraordinary mechanical properties of graphene. On the other hand, 
graphene is easy to bend in the out-of-plane direction. In reality, graphene samples exhibit many different distortions, such as ripples (Meyer et al., 2007) and folds (Kim et al., 2011). This aspect is advantageous for applications in flexible transparent electrodes and devices (Kim et al., 2009). Jeffy (2013) reported graphene to be one of the lightest and strongest first 2-D crystal material ever discovered having 0.77 milligrams per square meter weight. It has a spring constant in the range $1-5 \mathrm{~N} / \mathrm{m}$ and a Young's modulus of $0.5 \mathrm{TPa}$. It also demonstrates the hardest materials ever known and even harder than diamond and about 300 times than steel material. It is so flexible that it can be bent to different shapes or form and this makes it possible for used in ITO for screen guard application. It has a good thermal conductivity of about $3000 \mathrm{~W} / \mathrm{m}-\mathrm{K}$ in plane and about $2 \mathrm{~W} / \mathrm{m}-\mathrm{K}$ out of plane.

\subsection{Thermal Properties}

Graphene has an extraordinary thermal property dominated by the quantum of energy obtained when the energy of its lattice vibration is quantized and this makes it very attractive. Balandin et al. (2008) reported that graphene has extremely high in-plane thermal conductivity, about $5000 \mathrm{Wm}^{-1} \mathrm{~K}^{-1}$ at room temperature. This value is 10 times higher than that of copper with thermal conductivity $401 \mathrm{Wm}^{-1} \mathrm{~K}^{-1}$ and thereby making graphene far better than copper. This finding suggests graphene's high potential for use in microelectronics and for thermal management. According to Balandin (2011), heat conduction in carbon materials has been found to be dominated by phonons. This implies that as the number of graphene layers increases, cross-plane coupling of the low-energy phonons becomes dominant and significantly changes the phonon scattering processes. As a result, graphene's in-plane thermal conductivity decreases and reaches the value of graphite, $2000 \mathrm{~W} / \mathrm{mK}$, at around four layers (Balandin, 2011; Ghosh et al., 2010). Bao (2009) found graphene to have a negative thermal expansion coefficient given as $-6 \times 10^{-6} / \mathrm{K}$ approximately which is ten times larger than that of graphite. The large negative thermal expansion coefficient is due to out-of-plane phonons and is a direct consequence of the two-dimensionality of graphene (Singh et al., 2011).

\subsection{Optical Properties}

Graphene is characterized by its high mobility, conductivity and low absorption (2.3\%) of white light across the infrared and visible range spectrum. Though it may absorb some light at certain wavelengths, it would still be transparent to the naked eye. Since the Fermi level of graphene can be effectively tuned with electrostatic gating, the optical absorption can also be tuned electronically to further improve the transparency that makes it suitable for a wide range of industrial applications. According to Wang et al. (2008), the presence of a single-layer and AB-stacked bilayer has shown that the infrared absorption can be tuned with electrostatic gating effects.

Result from the experimental work of Geim and Novoselov in 2007 on transport measurements shows that at room temperature graphene exhibits a very high electron mobility in excess of $15,000 \mathrm{~cm}^{2} \mathrm{~V}^{-1} \mathrm{~s}^{-1}$ and the mobility of holes and electrons were found to be the same and explained reasons why defect scattering is the dominant scattering mechanism (Geim and Novoselov, 2007). Epitaxial graphene on $\mathrm{SiC}(0001)$ has been demonstrated to exhibit high mobilities on multilayered films at high temperature $\left(1000-1600^{\circ} \mathrm{C}\right)$. Kim et al. (2009) asserted that graphene deposited on polycrystalline $\mathrm{Ni}$ and transferred onto insulating substrates exhibit mobility values of up to $3650 \mathrm{~cm}^{2} \mathrm{~V}^{-1} \mathrm{~s}^{-1}$ and half-integer quantum Hall effect. Bonccorsoa et al. (2010) report on transmittance (T) and sheet resistance $\left(R_{s}\right)$ range that can be practically achieved for graphene layers of varying thickness and can be estimated by taking carrier density and charge mobility to be $\mathrm{n}=10^{12}-10^{13} \mathrm{~cm}^{-2}$ and $\mu=1,000-20,000 \mathrm{~cm}^{2} \mathrm{~V}^{-1} \mathrm{~s}^{-1}$ respectively, which is perculiar for films grown by CVD. However, the sheet resistance $\left(R_{s}\right)$ obtained in graphene is also achievable in ITO, $\mathrm{ZnO} / \mathrm{Ag} / \mathrm{ZnO}$ (Sahu et al., 2006), TiO2/Ag/TiO2 and SWNTs (Gaidau et al., 2016) with a similar or even higher optical transmittance. The best graphene-based transparent conductive films (TCFs) have been reported so far (Bae et al, 2010).

ITO has strong absorption above $4 \mathrm{eV}$ due to interband transitions Hamberg (1986), with other features at lower energy related to scattering of free electrons by tin atoms or grain boundaries.

Conductivity is based on ballistic electron transfer which is the transport of electrons in a medium with negligible electrical resistivity due to scattering resulting in high mobility. Figure 6 shows electron mobility of various materials, including graphene, graphene nanoribbons, carbon nanotubes, Ge(bulk), Si(bulk), SiMOS (Metal - Oxide - Semiconductor), III - V compounds (bulk) and 4H - SiC (bulk) (Schwierz, 2010). With the high charge mobility of graphene the introduction of bandgap by graphene nanoribbons could cause the charge mobility to suppress down to bulk phase of Si or even lower. 


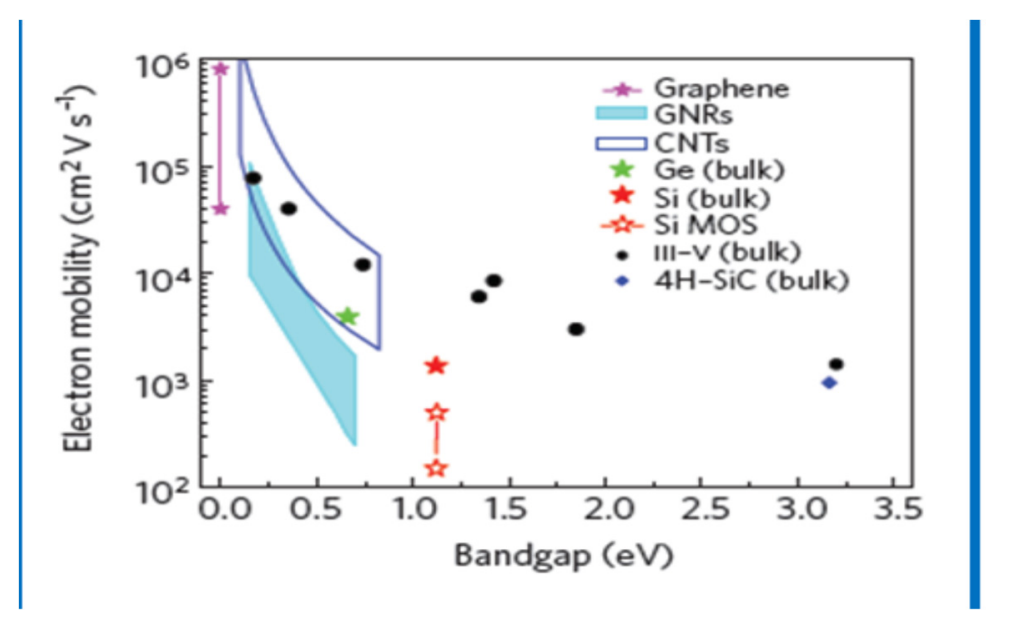

Figure 6. Electron mobility versus bandgap of various materials (Schwierz, 2010)

The charge mobility is theoretically limited to $\mu=200,000 \mathrm{~cm}^{2} \mathrm{~V}^{-1} \mathrm{~s}^{-1}$ by acoustic phonons at a carrier density of $\mathrm{n}$ $=10^{12} \mathrm{~cm}^{-2}$. The 2-D sheet resistivity also called the resistance per square, is then $31 \Omega$ by using the layer thickness we obtain a bulk conductivity of $0.96 \times 10^{6} \Omega^{-1} \mathrm{~cm}^{-1}$ for graphene, this is rather higher than the conductivity of copper which is $0.60 \times 10^{6} \Omega^{-1} \mathrm{~cm}^{-1}$.

\subsection{Electrical Properties}

Graphene is characterized by its high transparent nature (Transmittance), flexibility and low sheet resistance. Bonaccorso et al. (2010) investigation on the wide application of graphene in transparent conductors (optoelectronic devices) such as displays, touch screens, light-emitting diodes and solar cells require materials with low sheet resistance $\left(R_{S}\right)$ and high transparency $(T)$. This investigation assured the modification of the properties of graphene film by adjusting the sheet resistance of the material. Two methods were adopted by Li et al. (2009), Bae et al. (2010) \& Gunes et al. (2010) to reduce the value of the sheet resistance: layer-by-layer (LBL) and chemical/electrical doping.

LBL is the most useful method to reduce the value of sheet resistance by more than $50 \%$ but after four-layer transfer the reduction tends to saturate (Li et al., 2009a).

Updates in graphene studies has also shown the possibility of producing p-doped large area graphene sheet of 30 -inches with sheet resistance $\sim 30 \Omega \mathrm{sq}^{-1}$ and $\sim 90 \%$ optical transmittance at wavelength $\lambda=550 \mathrm{~nm}$. The study conducted by Bae et al. (2010) revealed that a low sheet resistance of $\sim 125 \Omega \mathrm{sq}^{-1}$ with $97.4 \%$ optical transmittance was obtained on the same size of graphene grown by CVD using flexible copper substrate. This result differs from the standard value of the sheet resistance by $24 \%$ with a slight difference in the optical transmittance showing a strong agreement with those of ITO that has sheet resistance range 10-30 $\Omega \mathrm{sq}^{-1}$, with optical resistance $90 \%$ using the same wavelength $(\lambda=550 \mathrm{~nm})$.

The use of the above method as investigated by Bae et al. (2010) confirmed the value of the sheet resistance to be superior to common transparent electrodes such as ITO and carbon nanotube films (Lee et al., 2008).

The use of chemical dopant methods can further improve the conductivity of graphene (Kang et al., 2011). SiC converted graphene over a large area has been reported and shown to exhibit outstanding electrical properties $(\mathrm{Wu}$ et al., 2009). Currently, indium tin oxide (ITO) mainly used for transparent conducting electrodes in various electronic devices, has proved to exhibit excellent sheet resistance of $10-50 \Omega \mathrm{sq}^{-1}$ at $\sim 90 \%$ optical transmittance in unlimited scalability (Bonaccorso et al., 2010) and (Hamberg \& Granqvist, 1986). Intrinsic single-layer graphene as reported by Bae et al. (2012) has a sheet resistance of $\sim 500 \Omega \mathrm{sq}^{-1}$ but most of the industrial applications require sheet resistance lower than the value used for transparent electrodes. In particular, the sheet resistance for organic light emitting diode displays, liquid crystal display; solar cells and electromagnetic interference shielding is less than $\sim 50 \Omega \mathrm{sq}^{-1}$, and for touch screens is $200-500 \Omega \mathrm{sq}^{-1}$.

Electrode of ITO at unlimited scalability commonly used in solar cells, touch sensors and flat panel displays has a sheet resistance of less than $100 \Omega \mathrm{sq}^{-1}$ with optical transmittance of $90 \%$. Previous investigation according (Kim, 2009; Cai et al., 2009), reported the best sheet resistance value to be $280 \Omega \mathrm{sq}^{-1}$ for graphene on nickel substrate by CVD on the centimeter scale. These features suggest the suitability of graphene for large area flexible ordinary light emitting diodes (OLEDs) (Kim et al., 2009; Chang et al., 2010). Also, a few atoms' thick graphene is 
mechanically compliant enough to be employed as an electrode for flexible OLED applications (Dikin et al., 2007; $\mathrm{Xu}$ et al., 2008). However, only few research groups have reported graphene OLEDs, of which power efficiencies $(<1 \mathrm{~lm} / \mathrm{W})$ are considerably low and emitting areas are small (Eda et al., 2008; Kim et al., 2009). Sheet resistance is calculated by (Royal et al., 2010).

$$
R_{S}=\frac{1}{\sigma}=\frac{1}{n e \mu}
$$

where $\sigma$ is the electronic conductivity, $\mu$ is the carrier mobility, $e$ is the electronic charge and $n$ is the number of concentration of electrons, respectively. It should be noted that the value of the transmittance from the spectra point of view or response of the human eye is highest at $550 \mathrm{~nm}$. Despite the numerous benefit of ITO, it still limited in it application such as; it skyrocketing price due to the short fall in the supply of indium element, processing requirements, difficulties in patterning (Hamberg, 1986 \& Granqvist, 2007) and a sensitivity to both acidic and basic environments. Furthermore, it is brittle in nature and can easily wear out or crack when used in applications involving bending, such as in touch screens and flexible displays (Sheraw et al., 2002). This implies that new transparent conductor materials are needed with improved performance. Recently, some materials were investigated and used as alternatives to transparent conductor materials, Metal grids (Lee et al., 2008b), metallic nanowires (De et al., 2009) and other metal oxides (Granqvist, 2007). From the reviewed applications and performance of transparent conductor materials nanotubes and graphene have shown great promise in becoming the future hope and better replacement for ITO. More so, graphene films have demonstrated a higher transmittance (T) over a wider wavelength range than single-walled carbon nanotube (SWNT) films (Geng et al., 2007), (Wu et al., 2004), (De \& Coleman, 2010), thin metallic films (Lee et al., 2008c) \& (De et al., 2009) and ITO (Hamberg, 1986 ) and (Minami, 2005). Interestingly, ITO is commercially available with transmittance $(\mathrm{T}) \approx 80 \%$ and sheet resistance (Rs) as low as $10 \Omega / \mathrm{sq}$ on glass (Minami, 2005) and $\sim 60-300 \Omega / \mathrm{sq}$ on polyethylene terephthalate (Granqvist, 2007).

\subsection{Gate Tuneable Band Structure in Few-Layer Graphene}

One of the most remarkable physical properties of graphene material is the ability to reversibly tune the band structure of these systems simply by means of an external electric field (Craciun et al., 2011)). In standard semiconducting materials a precise value of the bandgap is engineered during the growth process, therefore the value of this bandgap cannot be reversibly controlled in situ in a device. Few-layer graphene is the only known class of material to exhibit a gate tuneable band structure and this unprecedented property paves the way for devices with novel functionalities (Craciun et al., 2011).

\subsection{Work Function}

Work function is the energy which must be supplied to the free electrons in a metal to enable them to escape from the metal. The performance of an electronic device is measured by it work function such devices include: transistors (Yang et al., 2012), gas sensors (Janata \& Josowicz, 2003), solar cells (Page et al., 2014) and field emitters (Zhang, 2010). Bae et al. (2014) found work function $(\boldsymbol{\Phi}$ ) for self-assembled monolayer material-treated graphene films on $\mathrm{SiO}_{2}$ substrates to be 3.9, 4.25 and 4.5eV. This result confirms the effectiveness of the materials in controlling the graphene work fuction giving it opportunities for application in optoelectronic devices (Dibenedetto et al., 2009; Lee et al., 2010b). For a uniaxially strained graphene the work function increased with increasing strain. The experimental result (Oshima \& Nagashima, 1997) of the work function for graphene without strain is $4.5 \mathrm{eV}$ showing a strong agreement with the theoretical calculation (Giovannetti et al., 2008).

Bae et al. (2014) pointed out that the calculated values of graphene work fuction increases linearly. The work function became saturated at 5.2 and $4.8 \mathrm{eV}$ with $26 \% \mathrm{~A} / \mathrm{Z}$ strain. We can also engineer the work function of graphene films by varying the number of layers (thickness). When the thickness of one atomic layer changes it will affect a variations of several tens of meV (Hibino et al., 2009), (Yu et al., 2009), (Novoselov et al., 2012) and (Panchal et al., 2013). However, graphene work function is strained-induced and this can alter the device characteristics especially as it is dependent on the direction of the strain.

Zhou et al. (2016) found the work function of monolayer graphene to be $4.26 \pm 0.05 \mathrm{eV}$. The value increases to 4.60 $\pm 0.07 \mathrm{eV}$ for quad-layer graphene. Similarly, the graphene work function can also be extracted from the $\mathrm{He}+$ irradiated Secondary electron (SE) images obtained from scanning electron microscope (SEM). The work function varies from $4.35 \pm 0.02 \mathrm{eV}$ for monolayer to $4.56 \pm 0.03 \mathrm{eV}$ for quad-layer graphene. The two values of the work function for each layer are the same that is, there is no significant difference between the formal and the latter and 
this investigation is in agreement with previous investigations (Filleter et al., 2008, Hibino et al., 2009; Ziegler et al., 2011).

\section{Characterization}

\subsection{Structural and Morphological Property Characterization}

The structure and morphology of graphene on substrates could be investigated using raman spectroscope, scanning electron, transmission electron, atomic force microscopes, etc. Graphene sheet on $\operatorname{SiC}(000 \overline{1})$ surfaces characterized by Atomic force microscopy, Auger electron spectroscopy, scanning Auger microscopy and Raman spectroscopy according to Fisher et al. (2009) showed a flat morphology on $\operatorname{SiC}(000 \overline{1})$ and when compared with the graphene films grown on $\mathrm{SiC}(0001)$ surfaces it formed a terraced morphology. The difference in morphology is due to the contradictory interface structures in the two films. Also the morphology of graphene on the following substrates 001-GaAs, manganese p-doped 001-GaAs, and InGaAs were investigated by Stöberl et al. (2008) using scanning electron and atomic force microscopies and the result as compared with the layers on $\mathrm{SiO}_{2}$ shows that graphene sheets strongly follow the texture of the sustaining substrates but independent on doping, polarity, or roughness. The following techniques are used in the structural and morphological study of graphene.

\subsubsection{Raman Spectroscopy}

Raman spectroscopy has been extensively used as a nondestructive tool to investigate the structural and electronic characteristics of graphene according to Rao et al. (2009) and also analyze the number of layers in a sample in reality the quality of graphene is defined by the spectra from Raman spectroscopy. Recent investigation using Raman spectroscopy shows the $\mathrm{G}$ band peak to be higher than the $2 \mathrm{D}$ band peak indicating the presence a multi-layer graphene and this result is prevalent with nickel (Ni) catalyst. The $\mathrm{G}$ band peak being lower than the 2D band peak indicates few layer or single (mono) layer graphene common with the use of copper as catalyst Figure 7. The quality of sample is dependent on the $\mathrm{D}$ band peak which is the defect peak, the lower the peak the better the quality of the sample (Jeffy, 2013). The G band peak that is form as a result of the phonons in the Gamma Brillouin zone appears to be the most prominent band at $1580 \mathrm{~cm}^{-1}$ while the $2 \mathrm{D}$ band peak is due to the transverse optical phonons associated with K-Brillouin zones. Those formed a canonical structure with respect to graphene sheets according to $\mathrm{Ni}$ et al. (2008) shows that the 2D band's line shape and position depend on the number of layers and this represents the second order of zone boundary phonons which in turn do not satisfy the Raman fundamental selection rule. Naghdi et al. (2016) used X-ray diffraction (XRD), X-ray photoelectron spectroscopy (XPS) and Raman spectroscopy techniques to investigate the properties of graphene grown on Molybdenum (Mo) foil by CVD method at different growth temperatures $\left(1000^{\circ} \mathrm{C}, 1100^{\circ} \mathrm{C}\right.$, and $\left.1200^{\circ} \mathrm{C}\right)$.

The result from this investigation shows that the quality of the deposited graphene layers is affected by the growth temperature. However, as the growth temperature increases, the number of graphene layers and the number of defects related to the layers both decrease. It was also found that increasing the growth temperature increases the graphene domain size.

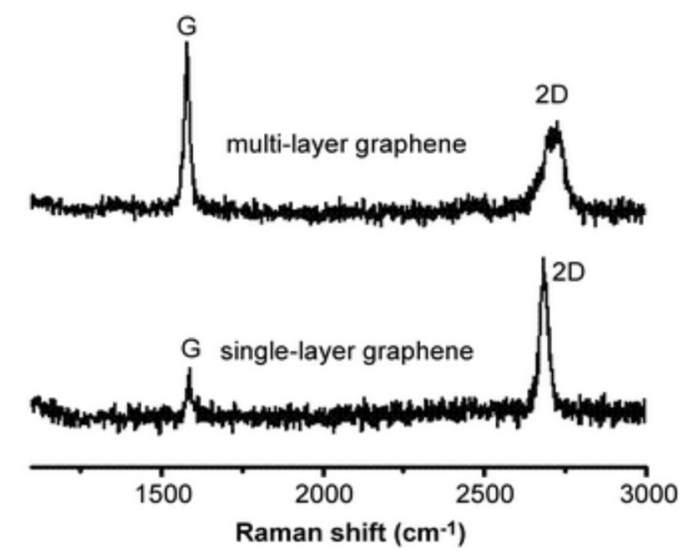

Figure 7. Typical Raman spectrum of multi-layer and single layer graphene (Yan \& Barron, 2010)

Further results from the Raman spectra of synthesized low temperature graphene as obtained in Jeffy (2013) show that, if the integral intensity ratio $2 \mathrm{D} / \mathrm{G}$ is less than 1 , then the material is multi-layer graphene, if greater than 1 , then it is a single-layer graphene and if equal to 1 , then the material is a two-layer graphene. The ratio between the 
$\mathrm{D}$ band peak and the $\mathrm{G}$ band peak is also important. If the integral intensity ratio $\mathrm{D} / \mathrm{G}$ is less than 0.5 , the sample is considered to be good quality otherwise in not a good film material.

\subsubsection{Atomic Force Microscopy}

Atomic force microscopy (AFM) measures the thickness of two-dimensional (2D) materials, predominantly graphene using the repulsive mode (Casiraghi et al., 2007). The thickness of the unreduced graphene oxide was found to be $\sim 1.0 \mathrm{~nm}$ and that of chemically reduced graphene oxide (rGO) $0.6 \mathrm{~nm}$. This difference in the thickness and the phase contrast arises from the hydrophilicity difference due to the distinct oxygen functional group in the reduction process. Previous research on graphene reveals that AFM has been exploited for mechanical and structural characterization of graphene because of it ability to feel the small force from the tip of the probe (Singh et al., 2011). In the future, new AFM modes will be developed and other modes for graphene are already suggested about mechanical, frictional, electrical, magnetic, and even elastic properties of graphene flakes.

\subsubsection{Scanning Electron Microscopy}

Scanning electron microscope (SEM) is one of the most important machines used to study the overall appearance of the grown sample. It determines the morphology/external topography and chemical composition of a specimen. Sample parameters like quality, shape, density, diameter, thickness, length and orientation of the as grown graphene are analyzed with SEM. SEM belong to the family of microscopes, but it uses a beam of electrons instead of light in order to make an image. The beam of electrons passes through the electromagnetic lenses and strikes the surface of the sample. The bombardment of electrons does not cause any damage to the samples. The detector collects the secondary/backscattered electrons ejected from the sample and converts them into a signal. Finally this signal is directed towards displaying screen (Zhou et al., 2007). SEM has the ability to capture the images in the range of visible to few nanometers, while the magnification range is around 20X-30000X along with a spatial resolution of $50-100 \mathrm{~nm}$. The electron acceleration voltages are normally in the range of $5-20 \mathrm{kV}$.

\subsubsection{X-ray Diffraction}

X-ray diffraction (XRD) is an analytical technique mainly used to identify the phases of crystalline materials (Cullity \& Stock, 2001) and other additional information such as lattice parameters and phase distribution using Rietveld confinement (Charl, 2015). Similar to the SEM set-up described in section 5.1.3, electrons are also produced from a cathode and accelerated to an anode target (commonly made of copper or cobalt) where bombardment takes place to produce $\mathrm{x}$-rays in a cathode ray tube. Depending on the anode material, characteristic $\mathrm{x}$-rays of different wavelength (for $\mathrm{Cu}, \lambda=1.5406 \AA$ or $\mathrm{Co}, \lambda=1.7890 \AA$ ) is produced. The XRD system is circular in nature and consists of the $\mathrm{x}$-ray tube, a sample holder and an $\mathrm{x}$-ray detector all lying on the focus circle. When the $\mathrm{x}$-rays interact with the sample, constructive interference occurs where Bragg's law is satisfied: that is;

$$
n \lambda=2 d \sin \theta
$$

where $\mathrm{n}$ is an integer value $(1,2,3), \lambda$ is the wavelength of the incident $\mathrm{x}$-ray, $\mathrm{d}$ is the spacing between the planes in the atomic lattice, $\theta$ is the angle between the incident ray and scattering plane. The angle between the projection of the $\mathrm{x}$-ray source and the detector is $2 \theta$. A detector records and processes the $\mathrm{x}$-ray signals and converts it to a count rate which is then conveyed to a monitor.

X-ray diffraction patterns of graphene oxide (GO), graphene (G), and graphene silver (Ag-G) nanoparticles hybrid materials were investigated by Sahu et al. (2013) and the pattern display of Graphene Oxide (GO) indicates a sharp diffraction peak at $2 \theta=14.03^{\circ}$ which is equivalent to (001) of GO figure 3.2. Specifically the display of G shows two distinct peaks at $2 \theta=24^{\circ}$ and $44.8^{\circ}$ respectively, which are attributed to (002) and (100) reflections of graphene nanosheets. The calculated d-spacing of graphene using broad peak was found to be $0.364 \mathrm{~nm}$. The peak tends to be very wide due to decrease in the crystallinity of graphene when reduced from graphene oxide. While the XRD pattern of silver graphene (Ag-G) reveals the peaks due to (111), (200), (220), and (311) of silver (Ag) nanocrystals as well as broad peak (002) due to graphene. Similar observation has been made for Au-G and $\mathrm{Cu}-\mathrm{G}$ nanohybrids but no peaks corresponding to oxides of $\mathrm{Ag}$, $\mathrm{Au}$, or $\mathrm{Cu}$ as were observed within the detectable limit of XRD. 


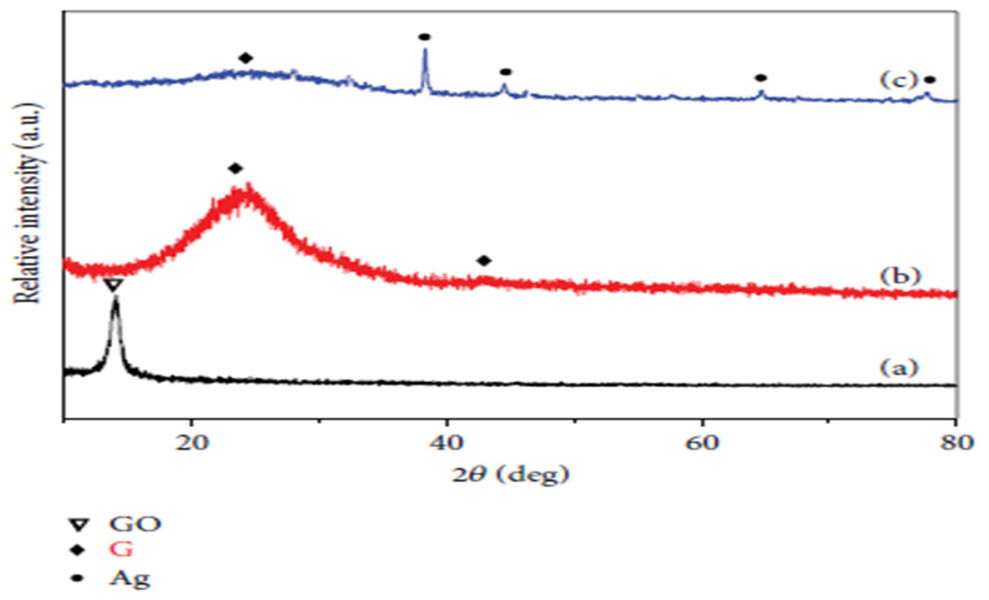

Figure 8. XRD patterns of: (a) GO, (b) G, and (c) Ag-G (Sahu et al., 2013)

\subsubsection{Transmission Electron Microscope}

Transmission electron microscope (TEM), like SEM, belongs to the family of electron microscopes. Sahu et al. (2013) investigation on microstructural characterization of graphene and graphene-metal nanoparticle hybrids using SEM and TEM result showed high resolution SEM micrograph of graphene that reveals crumbled and scrolled morphology of graphene sheets. TEM is a very valuable tool for the material's analysis and is used to obtain the structural information of a graphene at the atomic level. The working principle of TEM is just like optical microscope but with a relatively high energy electron source of around $200 \mathrm{KeV}$.

\subsubsection{X-Ray Photoelectron Spectroscopy}

$\mathrm{X}$-ray photoelectron spectroscopy (XPS) technique is used for the investigation of the surface composition in a sample and this helps to identify the surface recombination centers. XPS works better when the surface of the sample is illuminated by X-ray beam, such that the atom's core electron on the surface of the sample ingests whole $\mathrm{X}$-ray photon energy. The X-ray photon energy set electrons into vibration at minimum kinetic energy to produce photoelectron. This is shown by the relation between kinetic energy and photon energy expressed in equation 13 .

$$
\mathrm{E}_{\mathrm{k}}=\mathrm{h} v-\mathrm{E}_{\mathrm{b}}
$$

where $E_{\mathrm{k}}$ is the kinetic energy, $E_{b}$ is the binding energy and $h v$ is the X-ray photon energy.

Analyzed result from XPS shows bonding distribution in the grown sample through the intensities of each binding energy. For $\mathrm{sp}^{2}$ carbon to carbon hybridization the bonding of carbon is $284.8 \mathrm{eV}$ shifted from the oxidized defect in graphene oxide and effect indicates two different peaks of carbon bonds $\left(\mathrm{sp}^{2}\right)$ that decreases in intensity with plasma oxidation treatment for graphene grown via CVD. However, the CVD graphene is disposed to defect due it fabrication process and this is the reason for the single Gaussian peak in $\mathrm{sp}^{2}$ carbon Figure 9a.
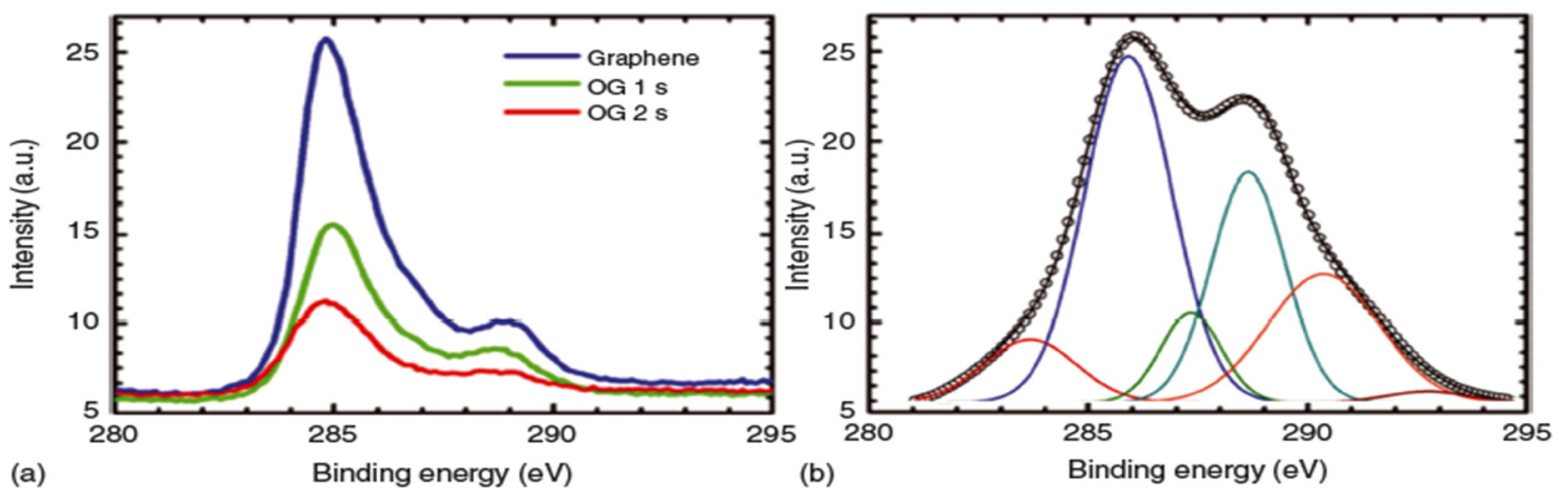

Figure 9. (a, b) XPS of graphene and graphene oxide (Jun, 2015) 


\subsubsection{Fourier Transform Infrared Spectroscopy}

Fourier transform infrared (FT-IR) spectroscopy is a non-destructive qualitative analysis technique used to determine unknown sample types by identifying the functional groups present in the sample. This investigation is done by exposing the sample to infrared (IR) radiation of which some part of it passes through (transmittance) the sample while some of it is absorbed (absorbance). A transmission, absorption or emission infrared spectrum is obtained over a wide spectra range expressed as wave numbers. FTIR spectra of all samples are usually recorded using a Bruker Vertex 77v FT-IR spectrometer which will be controlled using the Opus 7.0 spectroscopy software.

FT-IR measurements are then performed to determine the different vibration modes arising from the IR-excitation of the samples. Furthermore, this technique gives a good insight into the type of the interlayer anions present especially in layered double oxide materials (Yang et al., 2013). Measurements are performed within a wave number ranging from 300 to $3600 \mathrm{~cm}^{-1}$.

Lee et al. (2010a) pointed how significant the result from their analysis could contribute in revealing four main peaks in the FT-IR spectra of graphene Oxide (GO), the first peak at $1,050 \mathrm{~cm}^{-1}$ arises from epoxide groups $(\mathrm{C}-\mathrm{O}-\mathrm{C})$, the second peak which is the one centered at $1,680 \mathrm{~cm}^{-1}$ corresponds to the vibrational mode of the ketone groups $(-\mathrm{C}=\mathrm{O})$, the third peak at $1,380 \mathrm{~cm}^{-1}$ reveals the presence of a $\mathrm{C}-\mathrm{O}$ vibrational mode and the fourth the peak at $3,470 \mathrm{~cm}^{-1}$ shows $\mathrm{C}-\mathrm{OH}$ stretching Figure 10.(a and $\mathrm{b}$ ).
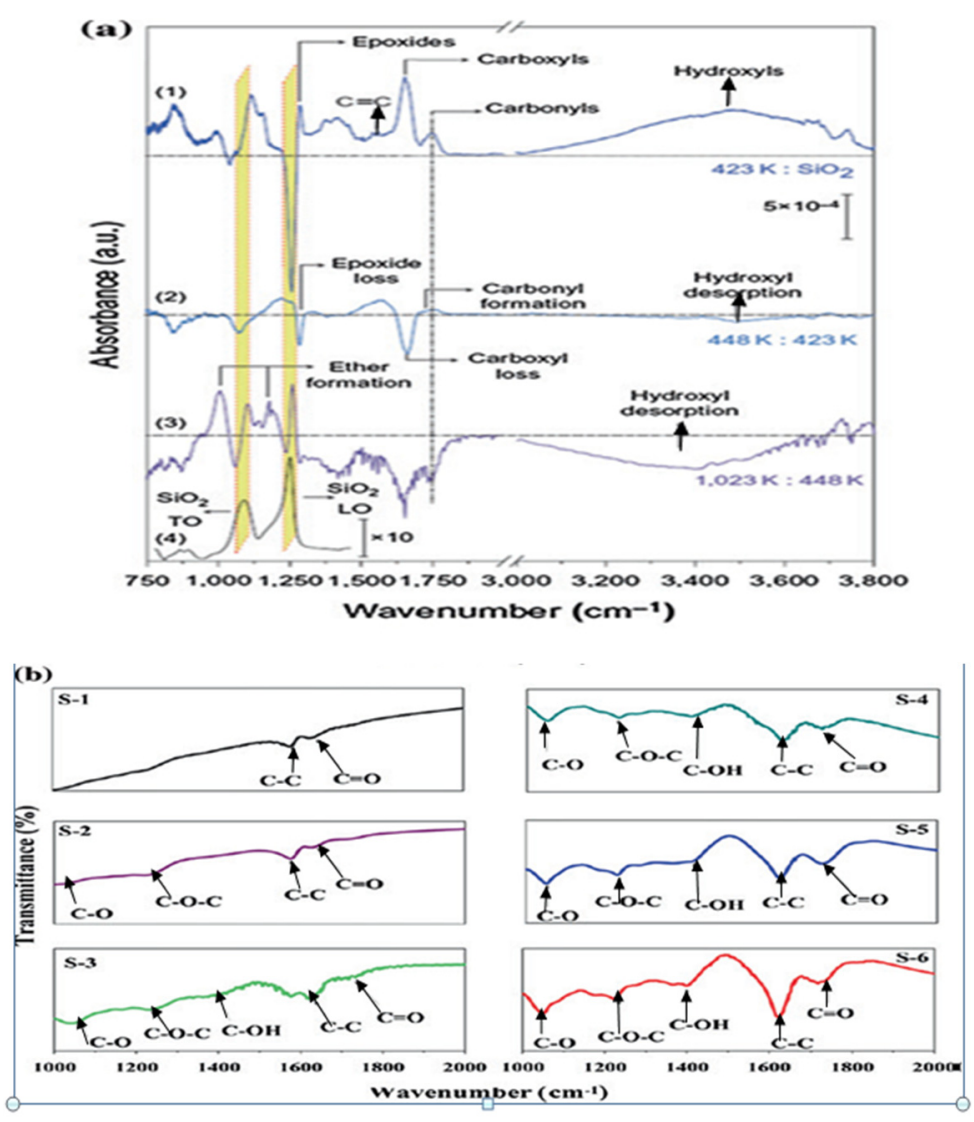

Figure 10. (a) FT-IR spectra of single-layer GO: annealing at $423 \mathrm{~K}$ and referenced to the bare oxidized silicon substrate the spectrum (1); annealing to $448 \mathrm{~K}$ (2), referenced to spectrum (1); annealing to 1,023K (3), referenced to spectrum (2); full $\mathrm{SiO}_{2}$ absorption of the oxide referenced to H-terminated silica (4) (Bagri et al., 2010); (b) FT-IR spectra of GO with different degrees of oxidation from samples S-1 to S-6 (Krishnamoorthy et al., 2013)

\subsection{Optical Property Characterization}

Here, the properties to be highlighted are transmittance (T), absorbance $(A)$, reflectance (R), optical bandgap (G), refractive index (n), dielectric constant, etc. of a graphene sample by Ultraviolet-Visible Spectroscopy ranging from different wave lengths in nanometer $(\lambda \mathrm{nm})$. 


\subsubsection{Ultraviolet-Visible Spectroscopy}

The optical properties of graphene film and graphene related hybrids are usually investigated using, Raman and UV-Visible Spectroscopy. From Avantes, UV-Visible Spectrophotometer has been used as an important tool for the characterization of nanoparticles to determine the Transmittance ( $T$ ), Absorbance rate and Reflectance of the sample. Investigation from the work of Tian et al. (2007) shows UV-Vis absorption spectra pattern for graphene oxide (GO), graphene (G), graphene-metal nanoparticle hybrids, Ag-G, Au-G, and Cu-G respectively. For GO, two peaks are noticeable in UV-Vis spectrum, one with a maximum at $230 \mathrm{~nm}$, due to $\pi \rightarrow \pi^{*}$ transition of aromatic $\mathrm{C}-\mathrm{C}$ bond and the other at $303 \mathrm{~nm}$, due to $\mathrm{n} \rightarrow \pi *$ transition of $\mathrm{C}=\mathrm{O}$ bond. Reduction of graphene oxide by hydrazine hydrate restore the electronic conjugation of the sample thereby promoting the formation of more absorption peak of $\mathrm{G}$ at the $266 \mathrm{~nm}$ while the second absorption at 303 disappears completely Figure 11 . The integration of Ag NPs, in the $\mathrm{G}$ framework (Ag-G) is confirmed by the presence of absorption peak at $420 \mathrm{~nm}$, which is considered to be due to surface plasmon resonance of Ag NPs.
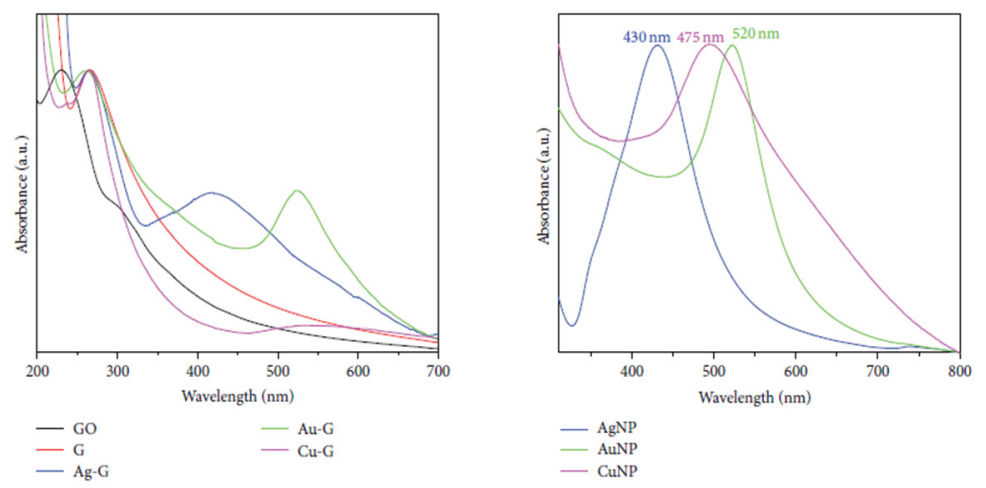

Figure 11. UV-VIS spectra of GO, G, Au-G, Ag-G, and Cu-G \& spectra of AgNP, AuNP, and CuNP (Sahu et al., 2013)

Table 2. Comparison of UV-Vis absorption peak position of GO, G, AgNPs, Ag-G, AuNPs, Au-G, CuNPs, and $\mathrm{Cu}-\mathrm{G}$ (Sahu et al., 2013)

\begin{tabular}{lll}
\hline Material & Graphene peak Position & Plasmon Peak Position \\
\hline Graphene Oxide (GO) & $230 \mathrm{~nm}\left(\pi \rightarrow \pi^{*}\right)$ & -- \\
& & - \\
Graphene (G) & -- & $430 \mathrm{~nm}$ \\
Pure silver nanoparticles (AgNP) & -- & $420 \mathrm{~nm}$ \\
Silver decorated graphene (Ag-G) & $260 \mathrm{~nm}$ & $520 \mathrm{~nm}$ \\
Pure gold nanoparticles (AuNP) & -- & $524 \mathrm{~nm}$ \\
Gold decorated graphene (Au-G) & $264 \mathrm{~nm}$ & $475 \mathrm{~nm}$ \\
Pure copper nanoparticles (CuNP) & -- & $540 \mathrm{~nm}$ \\
Copper decorated graphene (Cu-G) & $264 \mathrm{~nm}$ & \\
\hline
\end{tabular}

Jia et al. (2012) developed a method to optically detect graphene domains and domain boundaries directly on $\mathrm{Cu}$ foils through a simple selective annealing oxidation without further chemical treatments and transfer operations. This technique is simple and also allows for holistic considerations of all the factors for optimizing growth conditions and thus controlling graphene quality. Lack of detection technique for growth monitoring hinders deeper understanding of the factors that control graphene growth, such as graphene nucleation, temperature, gas feeding rate pressure (Jia et al., 2012), flow rate, heating rate and cooling rate of sample and oxidation effect.

The transparent nature of graphene allows the path of the visible light, which changes the interference color in contrast to the empty substrate. This can explain why graphene, even for a single-layer graphene can be visualized under an optical microscope by the naked eyes on silicon wafers with a certain thickness of $\mathrm{SiO}_{2}$ but only after multistep chemical treatments and complex transfer processes from growth substrates such as $\mathrm{Cu}$ foils Figure 12 . 


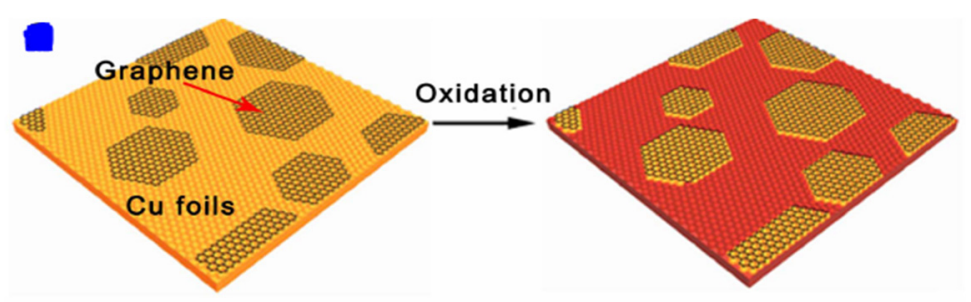

Figure 12. A schematic illustration of how to optically characterize graphene domains directly on $\mathrm{Cu}$ foils through selective oxidation (Liu et al., 2008)

Lui et al. (2008) reported the properties of graphene in retrospect to the thermal stability and impermeability of graphene which helps to protect the surface of $\mathrm{Cu}$ foils that is covered by graphene from oxidation. In disparity, due to the high chemical activity of $\mathrm{Cu}$, the bare surface of $\mathrm{Cu}$ foils is oxidized to form $\mathrm{Cu}$ oxides, thus leading to significant changes in colour. Colour change in $\mathrm{Cu}$ foils make graphene easily visible by the naked eyes using any common optical microscope for investigation as previously discussed but this does not serve a confirmatory test for the presence of graphene other characterization techniques are also needed. For better comparison, the pure $\mathrm{Cu}$ foils without graphene $(\mathrm{Cu})$, with partial graphene growth $(\mathrm{Cu} / \mathrm{pGr})$ and with full graphene growth $(\mathrm{Cu} / \mathrm{fGr})$ by a low pressure chemical vapor deposition (LPCVD) method at $1020^{\circ} \mathrm{C}$ are always used.

The results from this investigation were compared using both scanning electron microscopic (SEM) and optical methods to compare graphene morphologies on $\mathrm{Cu}$ substrates or $\mathrm{SiO}_{2} / \mathrm{Si}$ substrates after graphene transfer proving the reliability of the detection technique (Reina et al., 2008; Li et al., 2009a; Li et al., 2010 \& Liu et al., 2011).

\subsection{Electrical Property Characterization}

Electrical property of characterization involves the use of four-point probe also called "van der Pauw Method" to measure low resistivity of graphene.

\subsubsection{Four-Point Probe}

Four-point Probe is best used for a two dimensional sample. It has the ability to measure the properties of a sample of any arbitrary two-dimensional sample and has made this a very reliable method with a simple network. A current is sent to two probes and the voltage is measured through the other two. Then using Ohm's law, the resistivity can be calculated. This measurement is essential to determine how electrically conductive is the graphene sample when used as a transparent electrode (Vander-Pauw, 1958). The following properties of the sample can be determined from the use of this method they include: the resistivity, the doping type, the number of majority carriers per unit area, the mobility of the majority carrier.

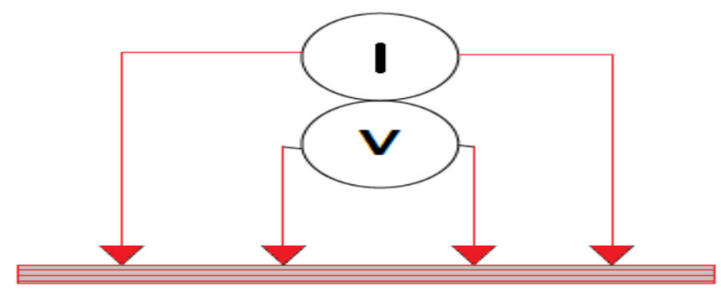

Figure 13. Four-point probing schematic

As shown in Figure 13, two probes are connected to a current source (I) and the voltage (V) is measured across the remaining two. But care must be ensured to remove the graphene surrounding the four contacts. This is to ensure correct measurement. Plasma etching is a popular method used to remove graphene. This is a drawback in this method as testing the resistivity of a large number of samples can make this expensive. All the samples cannot be tested using this technique as going through the procedure of removing some graphene can affect various other parameters and the usability of the sample.

\subsection{Mechanical Property Characterization}

This characterization involves the investigation of the Graphene's strength as it relates to many applications. Graphene samples have been found to be the strongest material ever tested Lee et al. (2008a) with an intrinsic 
tensile strength of $130 \mathrm{GPa}$ and a Young's modulus (stiffness) of 1TPa (150000000). The method involved in the mechanical characterization of graphene is called nanoindentation.

\subsubsection{Nanoindentation}

Nanoindentation technique is an alternative method modified to replace AFM technique. In this technique the cantilever used in AFM has been replaced with a nanoindentation device known as hysitron triboscope. This device is inserted on the material in order to obtain the hardness and elastic modulus of the material. It is for this reason that the method has been used for the characterization of the mechanical properties of graphene material (Wang et al., 2011).

\section{Applications and Uses of Graphene}

\subsection{Biomedical Application}

As applied in biomedical graphene enables a sensor system that can detect cancer cells and differentiate a single cancerous cell from a normal cell. It also encourages the fabrication of a simple noninvasive tool for early cancer diagnosis. According to Ray (2015), graphene system is able to detect the level of activity of an interfaced cell. The cell's interface with graphene rearranges the charge distribution in graphene, which modifies the energy of atomic vibration as detected by Raman spectroscopy in previous researches. Whenever a graphene structure is in contact with a cancer cell or a normal cell the atomic vibration energy in the structure changes immediately since the cancer cell's hyperactivity will always lead to a higher negative charge on its surface thereby enhancing the release of more protons. The electric field around the cell pushes away electrons in graphene's electron cloud, changing the vibration energy of the carbon atoms. The change in vibration energy can be detected by Raman mapping, allowing characterization of the activity of a single cell to take place. Graphene oxide (GO) or reduced graphene oxide (RGO) is used as biosensor for biosensing applications such as the detection of untimely disease and possibly suggest the cure for cancer patients. Graphene oxide has been used as an improved diagnostic for HIV patient. Graphene has been used as a fluorescence quenching material in biosensors that utilize the fluorescence resonance energy transfer (FRET) effect. Wang et al. (2010) used the FRET effect in a fluorescence in-labeled ATP aptamer to sense ATP as low as $10 \mu \mathrm{M}$. Lu et al. (2009) used single-stranded DNA (ssDNA) with a fluorescence tag and found that it bound noncovalently to $\mathrm{GO}$ with subsequent quenching of the fluorescence of the tag. Addition of a complementary ssDNA removed the tagged DNA from the GO surface and restored the fluorescence. Song et al. (2011) used folic acid-functionalized GO to detect human cervical cancer and human breast cancer cells.

\subsection{Graphene as a Battery}

Power is key to industrial growth and advancement in technology. Graphene has demonstrated extraordinary ability that made it extremely interesting and innovative material in portable energy storage devices. The used of batteries caught across many equipment particularly in automobiles (cars and motor bikes), aircraft, boats, ships, and electronic equipment (Zhang et al., 2008; Chen et al., 2012a; Chen et al., 2012b; Chen et al., 2012c). Lithium ion batteries have demonstrated the hub of energy source for conventional and promising energy storage devices that can be used in portable electronic applications (Chen et al., 2011). Recent focus on rechargeable batteries has made reasonable improvements in terms of higher capacity and compact size for convenient usage. Graphene and its composite materials were used as novel electrode materials for lithium-ion battery applications (Atabaki et al., 2013). The specific energy of $\mathrm{LiC}$ batteries is quite low $(370 \mathrm{mAh} / \mathrm{g})$ (Liang et al., 2009) because six carbon atoms can host only one lithium ion by forming an intercalation compound $\left(\mathrm{LiC}_{6}\right)$.

\subsection{Transparent Electronics}

\subsubsection{Graphene Thin Film as Transparent Conductor (Electrodes)}

Graphene is an elastic/flexible thin film that behaves like transparent plastic; it conducts heat and electricity better than any metal. It behaves like an impermeable membrane and is chemically inert and stable. Thus, graphene is proven to be material for the production of the next generation transparent conducting electrodes required for different applications such as solar cells, light-emitting diodes (LEDs), organic light-emitting diodes (OLEDs), touch screens, smart windows and liquid crystal displays (LCD), and organic photovoltaic cells (OVPs) (Bonaccorso et al., 2010). There is need to find a substitute for indium tin oxide (ITO) in the manufacturing of various types of displays and touch screens due to the scarcity of indium and its consequent growing cost. In particular, graphene's mechanical strength and flexibility are advantageous compared with ITO that is brittle. Therefore, abundance of carbon on earth made graphene a more sustainable alternative for these applications. 


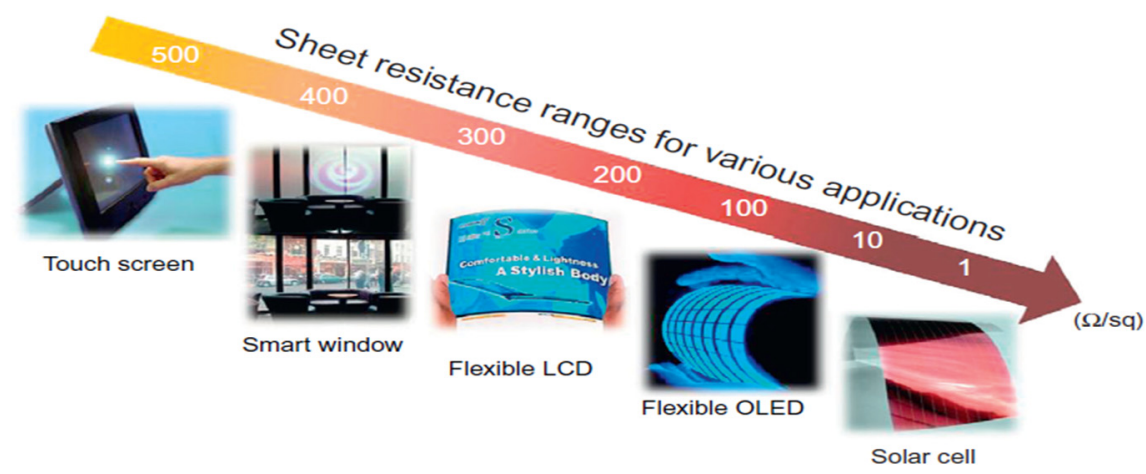

Figure 14. Potential graphene applications and corresponding resistance range required for each application (Bae et al., 2012)

\subsubsection{Flexible Electronics}

Graphene and graphene-related 2D crystals and hybrids will have a disruptive impact on current optoelectronics devices based on conventional materials, not only because of cost/performance advantages but also because they can be manufactured in more flexible ways suitable for a growing range of applications such as touch screens and flexible displays.

\subsubsection{Touch Screen}

Touch screen is another potential application of graphene transparent conductive films that have been adopted in various electronic devices such as cell phones, laptops, smart phones, e-books, and others. Researchers in Korea and Japan have fabricated films of graphene-planar sheets of carbon that are one atom thick-measuring tens of centimeters. The researchers engineered these large graphene films into transparent electrodes, which were incorporated into touch screen panel devices. Different types of commercialized touch screens are available today in the market. Resistive-type touch screens are operated through induction of an electric short between top and bottom transparent conducting films. They required a sheet resistance of up to $550 \Omega / \mathrm{sq}$ and an optical transmittance of more than $90 \%$ at $550-\mathrm{nm}$ wavelength. Typically, ITO films have been widely used in these applications.

Bae et al. (2010) reported layer-by-layer graphene stacking to fabricate a doped fourlayer graphene thin film on

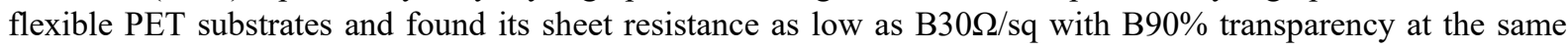
wavelength, which is superior to commercial transparent electrodes such as ITO that could be used in a fully functional touch screen panel device capable of withstanding high strain. In this fabrication, all of the materials are flexible and the screen operates reliably after many bending cycles. In addition, capacitive touch screens fabricated using transparent graphene films are also available. Lee \& Ahn (2013) demonstrated that graphene films can satisfy the requirement of a sheet resistance of $100 \Omega / \mathrm{sq}$ through doping, this investigation predicted that in the future, transparent graphene films would replace rigid and brittle ITO films in touch screen electrodes.

\subsubsection{Solar Cells and OVPs}

Graphene is both highly conductive and transparent. As such, it has great potential as a material in solar cells. Solar cells like polymer, bulk hetero-junction, and dye-sensitized are the most important promising devices for the conversion of sunlight into electrical energy (Bundgaard et al., 2010).

They also offer the advantages of low cost and large-scale production i.e., the smaller the material size the lower the cost of production. From the practical point of view rapid growth of the photo voltaic (PV) industry causes the depletion of the raw materials involved in the production of solar panels. Typically, solar cells use silicon, which produces a charge when a photon hits the materials, knocking loose a free electron. Silicon only releases one electron per photon that hits it. Previous research on graphene has shown that graphene structure can release multiple electrons for each photon that hits it and this characteristics makes it far better at converting solar energy, with a projected 60 percent efficiency compared to the roughly $25 \%$ efficiency that current silicon cells are capable of. Unfortunately, this is all theoretical. Current graphene cells are not yet up to par with silicon cells. Currently research on graphene solar cells across the world is aimed at increasing the efficiency of the solar cells. In no distance time, cheaper, more powerful graphene cells that will produce a massive surge in renewable energy would 
be in the market. Most of the solar cells are ITO with a nonconductive glass protective layer to meet their needs. OPV devices are made of an organic layer sandwiched between two charge-collecting electrodes, one of which must be transparent (e.g., ITO or fluorine tin oxide (FTO)) and the other is usually aluminum, sometimes coated with $\mathrm{LiF}$ or $\mathrm{MgO}$. Efforts were made to replace these materials by finding alternative electrode materials for the fabrication of solar cell applications. However, ITO is a nonflexible material and graphene and graphene-based nanocomposite are not only flexible but also extremely conductive and could be very useful in solar cell devices. Wu \& Zheng (2013) achieved photoelectron conversion efficiency of B7.5\% using graphene nanosheets (GNSs) incorporated with activated carbon as the counter electrode. Molybdenum sulfide $\left(\mathrm{MoS}_{2}\right) /$ graphene composite electrodes have been used as a counter electrode for dye-sensitized solar cells (DSSCs) (Yue et al., 2012). The composite-modified electrode has higher current density than those of $\mathrm{MoS}_{2}$. This composite exhibited a power efficiency value of $5.98 \%$, which was comparable with that of the Pt electrode $(6.23 \%)$, and revealed good performance of the molybdenum-based composite electrode. The fabrication of low-cost DSSCs achieves high efficiency.

\subsubsection{Organic Light-Emitting Diodes (OLED)}

An important component of organic light-emitting diode (OLEDs) is the anode as transparent electrode material, injecting charge carriers and allowing light to pass through. Graphene films are attractive materials for flexible transparent conductive electrodes in OLEDs due to their controllable transparency, good electrical conductivity, and suitably tunable work function. In particular, graphene films have a molecular structure similar to that of organic electronic materials, and thus can form strong bonds with organic electronic materials (Wu et al., 2010; Han et al., 2012; Sun et al., 2010; Hwang et al., 2012).

Many OLEDs based on CVD graphene films have been reported (Sun et al., 2010; Hwang et al., 2012). Among them, Han et al. (2012) fabricated OLED devices based on CVD graphene films deposited on copper foils. They have observed that the OLEDs have extremely high performance compared with those based on conventional ITO (Han et al., 2012). In this case, graphene films transferred onto PET substrates that act as anode material were controlled in terms of electrical resistance, optical transmittance, and work function by random piling of multiple layers and acid doping (Han et al., 2012). The sheet resistance and work function varied from 89 to $189 \mathrm{Ohm} / \mathrm{sq}$ and 4.33 to $4.45 \mathrm{eV}$ as a function of the number of layers.

\subsubsection{Liquid Crystal Displays}

Graphene has high electrical conductivity and excellent transmittance at terahertz frequencies, so $\mathrm{Wu} \&$ Zheng (2013) and Wu et al. (2013) demonstrated a liquid crystal-based terahertz phase shifter with this graphene. They observed in a 50- $\mu \mathrm{m}$ liquid crystal cell that the maximum phase shift is 10.8 with saturation voltage of $5 \mathrm{~V}$. This phase shifter provides continuous tunability, fully electrical controllability, and low DC voltage operation that depend on the number of graphene layers.

Blake et al. (2008) also demonstrated liquid crystal devices with electrodes made of graphene that show excellent performance with a high contrast ratio that can be comparable with conventionally used metal oxides in terms of low resistivity, high transparency and chemical stability.

\subsection{Integrated Circuits}

For integrated circuits, graphene has high carrier mobility as well as low noise, allowing it to be used as the channel in field effect transistors (FETs). Single sheets of graphene are difficult to produce and even more difficult to make on an appropriate substrate (Chen et al., 2007). Ponomarenko et al. (2008) fabricated the smallest transistor so far using graphene that is one atom thick and 10atoms wide. Wang et al. (2009a) fabricated an n-type transistor, meaning that both $\mathrm{n}$-type and $\mathrm{p}$-type graphene transistors had been created. A functional graphene integrated circuit was demonstrated a complementary inverter consisting of one p-type and one n-type graphene transistor (Traversi et al., 2009). However, this inverter suffered from very low voltage gain. Lin et al. (2010) grew epitaxial graphene on $\mathrm{SiC}$ with quantity and quality suitable for mass production of integrated circuits.

The first graphene-based integrated circuit was fabricated with a broadband radio mixer, and the circuit handled frequencies up to $10 \mathrm{GHz}$. Its performance was unaffected by temperatures up to $127^{\circ} \mathrm{C}$ ( $\mathrm{Lin}$ et al., 2011).

\subsection{Radio Frequency Applications}

The development of transparent radio frequency electronics has been limited, until recently, by the lack of suitable materials. Naturally, thin and transparent graphene may lead to disruptive innovations in such applications. Wu et al. (2014) realized optically transparent broadband absorbers operating in the millimeter wavelength regime achieved by stacking graphene bearing quartz substrates on a ground plate. Broadband absorption is a result of mutually coupled Fabry-Pérot resonators represented by each graphene quartz substrate. Millimeter wave 
reflectometer measurements of the stacked graphene -quartz absorbers demonstrated excellent broadband absorption of $90 \%$ with a $28 \%$ fractional bandwidth from 125 to $165 \mathrm{GHz}$ bandwidth. This result suggests that the absorber operation could be extended to microwave and low terahertz bands with negligible loss in performance. The transparent material could be used as a coating for car windows or buildings to stop radio waves from traveling through the structure and to improve secure wireless network environments.

\subsection{Fuel Cells}

Fuel cells are a type of energy storage device that converts chemical energy from a fuel into electrical energy by using oxygen and methanol. The green energy of fuel cells could be obtained from reduction of oxygen (Qu et al., 2004) and oxidation of methanol (Golabi et al., 2002). Most of the electrocatalytic performance is based on their selection in suitable electrode materials. Numerous efforts were made in the literature for the utilization of carbon-based composite materials toward oxygen reduction reactions (Wu et al., 2011). One of the main issues connected with fuel cells is the limited availability of platinum (Pt), which is a candidate catalyst for fuel cell reactions. After the discovery of graphene, it was found to have widespread applications in fuel cells, where it can be used as an excellent electrode material because of its excellent physicochemical properties. Graphene has a high surface area, making it more efficient than carbon black for dispersing Pt nanoparticles (Xin et al., 2011).

\subsection{Graphene Quantum Dots}

All dimensions of graphene quantum dots (GQDs) are 10nm. Their size and edge crystallography govern their electrical, magnetic, optical, and chemical properties. GQDs can be produced through graphite nanotomy (Mohanty et al., 2012) or via bottom-up, solution-based routes (Cai et al., 2010). GQDs with controlled structures can be incorporated into applications in electronics, optoelectronics, and EMs. Quantum confinement can be created by changing the width of GNRs at selected points along the ribbon (Ponomarenko et al., 2008).

\subsection{Frequency Multiplier}

In 2009, researchers built an experimental graphene frequency multiplier that takes an incoming signal of a certain frequency and produces a signal as a multiple of that frequency at the output (Wang et al., 2009; Wang et al., 2009; Wang et al., 2009; Cricchio et al., 2009).

\subsection{Optical Modulator}

When the Fermi level of graphene is tuned, its optical absorption can be changed. In 2011, Liu et al. reported the first graphene-based optical modulator. This modulator operates at $1.2 \mathrm{GHz}$ without a temperature controller and has a broad bandwidth (from 1.3 to $1.6 \mu \mathrm{m})$ and small footprint $\left(\mathrm{B} 25 \mu \mathrm{m}^{2}\right)$.

\subsection{Piezoelectricity}

Density functional theory simulations predict that depositing certain adatoms on graphene can render it piezoelectricity responsive to an electric field applied in the out-of-plane direction. This type of locally engineered piezoelectricity is similar in magnitude to that of bulk piezoelectric materials and makes graphene a candidate for control and sensing in nanoscale devices (Ong \& Reed, 2012).

\subsection{Graphene as Purification of Water}

Graphene sheets perforated by small holes were first explored as potential candidates for water filtration by researchers at MIT. Holes with a diameter of 1nanometer (a billionth of a meter) are big enough to let water molecules sift through, but they are small enough to stop any undesired chemicals. Han et al. (2013) fabricated ultrathin ( $22-53 \mathrm{~nm}$ thick) graphene nanofiltration membranes (uGNMs) on microporous substrates that were used for efficient water purification. The performance of the uGNMs for water treatment was evaluated on a dead-end filtration device, and the pure water flux of uGNMs was high $(21.8 \mathrm{~L} / \mathrm{m} 2 / \mathrm{h} / \mathrm{bar})$. The uGNMs showed high retention (> 99\%) for organic dyes and moderate retention $(\sim 20-60 \%)$ for ion salts. Graphene membrane for water purification is the best so far because of its ability to filter out all carbonic, ionic and impurity substances.

\section{Advances and Future Prospects}

\subsection{Future Technology}

The following questions are meant to forecast the future and prospects in graphene research.

So where will graphene take us? How will it change our world? What benefits will it bring to mankind? What applications will we see in the near future and decades to come? Clean drinking water for millions. Graphene membranes could see huge progress in water purification technology in developing countries and provide more efficient desalination plants. Electronics and energy storage ar band to be revolutionized by graphene. Flexible, durable, semi-transparent mobile phones, wearable technology, clothing that communicates will be made available 
for use. Electric sports cars, light weight planes all these are the future technologies which are becoming realistic in our present time.

\subsubsection{Recent Application in Graphene Research}

Researchers integrate graphene and quantum dots with CMOS technology to create an array of photodetectors, producing a high resolution image sensor.

\subsection{Future Work}

To achieve the ambitious goal of developing new materials like graphene enabled electronic devices, the following growth-related issues still have to be overcome: the transfer process has to be made more reliable and reproducible; the sample to sample variation introduced by handling the copper substrate and the membrane is currently a large factor and the inability to control the sample quality. Furthermore, more studies should be carried out to identify changes made to the graphene material during the transfer process in terms of doping, introduction of defects, etc.

The lowering of the growth temperature could have benefits for the compatibility with other materials and processes and could also decrease the impact of cooling induced wrinkling on the morphology of graphene. The growth on dielectric substrates would resolve many problems associated with metallic growth substrates, such as the need for a transfer step, large thermal mismatch between substrate and graphene, etc. Better control of both the formation and the extension of additional layers on the graphene through substrate engineering are crucial. In this regard, several approaches of graphene application could be envisaged in the following:

(1) More complex circuits on flexible substrates or complex surfaces should be designed.

For example, a display could be produced by using commercially available electroluminescent or electrochromic materials in a sandwich structure between graphene layers.

(2) The resolution limits of the passivated growth technique should be explored to identify whether high quality nanostructures could be synthesized and other passivation materials could be explored to describe area-selective for CVD process that can be more easily etched.

(3) A comparative study of capacitance, cyclic stability, increase in energy storage density and the overall battery performance should be investigated using graphene as a single and both electrodes respectively. i.e. at both cathodes and anodes simultaneously. Future researchers can also investigate if adding more graphene at either electrode can yield added benefits or the pertinent drawbacks would outweigh the benefits.

(4) A more detailed study of the sensor characteristics (including a detection limit) of the films in relation with their synthesis conditions should be the subject of future investigations. Future research should focus on replacing graphene with silicon in electrical systems and telecommunication devices.

\subsection{The Present Challenges In Graphene Research}

The exceptional properties of graphene, including electrical, thermal, mechanical, optical, and long electron mean free paths, makes it compelling for various engineering applications. Huge efforts have been devoted to the fundamental physics and chemistry of graphene. Properties such as room temperature quantum hall effect, highest charge transport, and thermal conductivity originated from graphene's 2-D structures have not been observed from most conventional 3-D materials. Several research publications in the past 10 years signify the importance of graphene and that it might surpass silicon research in the development of microelectronics. Although silicon-based research is at a mature stage to overcome technological barriers, graphene is being extensively investigated because it holds the future for microscale to nanoscale electronics. The inherent semi-metal characteristic of graphene has been modified to realize the applications in transistors. Much effort has been devoted to modification of a graphene bandgap, allowing applications in electronic devices. Graphene as a new material still faced many challenges ranging from synthesis and characterization to the final device fabrication. The exceptional properties were observed in the defect-free pristine graphene prepared by graphite exfoliation using the scotch tape method, which is not appropriate for any large-scale device manufacturing. Alternative methods have progressed to CVD and epitaxially grown single-layer, bilayer, and few-layer graphene on different substrates and simple transfer of the graphene layer for subsequent device fabrication. These breakthroughs will offer novel and exciting opportunities for semi-conductor industries. The advanced deposition technique of the single layer and bilayer made it possible to fabricate large-area devices; however, creating bandgap in a controlled and practical manner is still challenging for application in logic devices. Several methods aimed to alter the substrate properties and nanoribbon dimension have been proposed to introduce a tunable bandgap essential for nanoelectronics. This energy bandgap can be achieved through quantum confinement, bilayer graphene, and chemical functionalization. The former, quasi-1D GNR has been considered with either edge localization or Coulomb blockade effects. GNR 
with appropriate dimensions (i.e., 10nm) is expected to provide right bandgap for efficient FET devices. Therefore, the bandgap opened graphene based on GNR has been encouraged for future practical nano electronic devices comparable with complementary metal oxide semiconductor (CMOS) circuits. Perhap, the greatest challenge is that, although graphene as vital as it is to our future that it has been named a "supply critical mineral" and a "strategic mineral" by the united states and the European Union, only a few companies around the world have access to the mineral that is required to make graphene and $70 \%$ of the supply is control by China.

\section{Conclusion}

1. The assurance of using of silicon in modern technology is becoming problematic as it produces undesired gain without the integrated circuit (IC). This challenge requires a rethink on how to manufacture devices, or even whether we need an alternative to silicon itself. The performance of silicon degrades progressively at high temperatures and becomes very poor at transmitting light and this indicate the limit of its application and therefore quest for a better replacement. The discovery of graphene with its unique nano-scale properties is gradually making the way to possible substitutes for the next generation of faster and smaller electronics in the last decade.

2. Rise in demand and production level as the world population increases and convenience in the use of two-dimensional (2D) materials would lead to the extensive use of graphene made materials in a wide variety of applications. Products made of graphene are attracting serious attention in the market. As a result of the promising properties of graphene, the research in the field is also attracting large grants and sponsors with an incremental rise in the number of papers as highlighted in the trend in graphene research early in this paper.

3. The lack of band gap in graphene material is based on the fact that the unit cell of graphene has two atoms with the same potential. The development of graphene based electronics depends strongly on our ability to open a tunable bandgap. So, bandgap is introduced by making the potential of one atom higher than the other by the application of an electric field.

Although several attempts have been made by patents as revealed in the literatures using different approaches to fabricate high performance graphene devices and consequently engineer the bandgap so as to improve their semiconducting properties, these attempts still linger. Investigation from theoretical and computational analysis proved to have solved the zero bandgap problem of graphene while we wait for a convincing approach that will break this dilemma.

4. To generate a bandgap, one has to make the two atomic sites have different potentials which is quite difficult given that they are 0.15 nanometer apart. Another approach is to reduce the dimension of graphene from 2-D to 1-D i.e make a nanoribbon of the graphene. This opens a bandgap through the 1-D confinement of the electrons.

5. Among the methods employed to initiate a bandgap for nanoelectronics applications subtrate effect, reduction in the dimensions as in nanoribbon and electric field application are considered most important. In addition, the choice of Nitrogen (N) and Boron (B) as substitute to carbon atoms that form carbon alloys is another way of creating a bandgap in graphene. Creating a bandgap in graphene using bilayer instead of monolayer would in principle, lead to a lower intrinsic carrier mobility.

6. We trust that graphene would provide a greater opportunity for the development of high performance electronic devices having shown great potential in the field of transparent conductive electrodes.

7. Despite the advances in graphene research, obtaining a controlled method on the film size which can produce larger crystalline graphene domains is still not available this calls for improvement on graphene growth.

8. Graphene film grown using camphor precursor shows superior quality than that of the film grown using methane at low temperature. Results revealed that camphor precursor is a good alternative to hydrocarbon precursors for graphene research. The oxygen present in the camphor molecule is seen to be beneficial for both controlling the thickness and passivating $\mathrm{Cu}$ substrate during growth.

9. The properties of graphene-like films are influenced by the synthetic conditions as well as the electrical resistance which depends on the ambient conditions.

10. We can improve the quality of graphene by reducing the thickness of the catalyst used for the growth. For example, copper and nickel can be reduced in thickness. The results obtained suggest that the proposed method is highly promising for obtaining carbon films, which possess a selective sensitivity to ethanol vapour.

\section{Conflict of interests}

The authors declare that there is no conflict of interests regarding the publication of this paper. 


\section{References}

Abergel, D. S. L., Russell, A., \& Falko, V. I. (2007). Appl. Phys. Lett., 91, 063125.

Acheson, E. G. (1896). United States Patent (Vol. 568323).

Addou, R., Dahal, A., Sutter, P., \& Batzill, M. (2012). Monolayer graphene growth on Ni (111) by low temperature chemical vapor deposition. Applied Physics Letters, 100(2), 021601-021603.

Amarnath, C. A., Hong, C. E., Kim, N. H., Ku, B.-C., Kuila, T., \& Lee, J. H. (2011). Carbon, 49, 3497.

An, H., Lee, W., \& Jung, J. (2011). Graphene synthesis on Fe foil using thermal CVD. Current Applied Physics, $11, \mathrm{~S} 81-\mathrm{S} 85$.

Arsem, W. C. (1911). Ind. Eng. Chem., 3, 799.

ASM Handbook; ASM International. (2002). Alloy Phase Diagrams (Vol. 3). Retrieved from https://en.wikipedia.org/wiki/Raman_scattering date of retrieved 28/12/2016; $\mathrm{http} / / /$ theconversation.com/with-silicon-pushed-to-its-limits-what-will-power-the-next-electronics-revolutio n- 46287

Atabaki, M. M., \& Kovacevic, R. (2013). Graphene composites as anode materials in lithium ion batteries. Electron Mater Letter., 2, 133-153.

Atta, N. F., Galal, G., \& El-Ads, E. H. (2015). A Platform for Sensor and Biosensor Applications.

Bae et al. (2010). Roll-to-roll production of 30-inch graphene films for transparent electrodes. Nat. Nanotechnol., 5, 574-578.

Bagri, A., Mattevi, C., Acik, M., Chabal, Y. J., Chhowalla, M., \& Shenoy, V. B. (2010). Nat. Chem., 2, 581-587.

Balandin, A. A. (2011). Thermal properties of graphene, carbon nanotubes and nanostructured carbon materials. Nat. Mater., 10(8), 569-581.

Balandin, A. A., Ghosh, S., Bao, W. Z., Calizo, I., Teweldebrhan, D., Miao, F., \& Lau, C. N. (2008). Superior thermal conductivity of single-layer graphene. Nano Lett., 8, 902-907.

Balog et al. (2010). Nat. Mater., 9, 315.

Bao, W. Z., Miao, F., Chen, Z., Zhang, H., Jang, W. Y., Dames, C., \& Lau, C. N. (2009). Controlled ripple texturing of suspended graphene and ultrathin graphite membranes. Nat. Nanotech., 4, 562-566.

Blake et al. (2008). Graphene-based liquid crystal device. Nano Lett., 8, 1704-1708.

Boehm, H. P., Clauss, A., \& Hofmann, U. (1962). Dünnste Kohlenstoff-Folien. Zeitschrift für Naturforsch., 70(b), $150-153 \mathrm{~s}$.

Bonaccorso, F., Sun, Z., Hasan, T., \& Ferrari, A. C. (2010). Graphene photonics and optoelectronics. Nat Photon, 4, 611-622.

Brenner, D. W., Shenderova, O. A., Harrison, J. A., Stuart, S. J., Ni, B., \& Sinnott, S. B. (2002). A second-generation reactive empirical bond order (REBO) potential energy expression for hydrocarbons. J. Phys. Condens. Matter, 14(4), 783-802.

Bundgaard, E., Hagemann, O., Manceau, M., Jorgensen, M., \& Krebs, F. C. (2010). Low BandGap Polymers for Roll-to-Roll Coated Polymer Solar Cells. Macromolecules, 43(19), 8115-8120.

Cai et al. (2010). Atomically precise bottom-up fabrication of graphene nanoribbons. Nature, 466(7305).

Casiraghi, C., Pisana, S., Novoselov, K. S., Geim, A. K., \& Ferrari, A. C. (2007). Raman Fingerprint of Charged Impurities in Graphene. Applied Physics Letters, 91(23), 233108.

Castro-Neto, A. H., Guinea, F., Peres, N., Novoselov, K. S., \& Geim, A. K. (2009). The electronic properties of graphene. Rev. Mod. Phys., 81(1), 109-162.

Chang, H., Wang, G., Yang, A., Tao, X., Liu, X., \& Shen, Y. (2010). Adv. Funct. Mater, 20(17), 2893.

Charl, J. J. (2015). Synthetic Strategies to Improve the Performance of Manganese Oxide Based Layered and Spinel Materials for Electrochemical Energy Storage. Thesis (PhD) University of Pretoria.

Charlier, J. C., Michenaud, J. P., \& Gonze, X. (1992). First-principles study of the electronic properties of simple hexagonal graphite. Phys. Rev. B, 46(8), 4531-4539. 
Chen, C. Y., Rosenblatt, S., Bolotin, W., Kalb, P., Kim, I., Kymissis, H. L., Stormer, T., Heinz, F., \& Hone, J. (2009). Performance of monolayer graphene nanomechanical resonators with electrical readout. Nat. Nanotech., 4, 861-867.

Chen, D., Mei, X., Ji, G., Lu, M., Xie, J., Lu, J., \& Lee, J. Y. (2012). Reversible lithium_ion storage in silver-treated nanoscale hollow porous silicon particles. Angew. Chem. Int. Ed., 51, 2409-2413.

Chen, J. H., Ishigami, M., Chaun Jang, C., Hines, D. R., Fuhrer, M. S., \& Williams, E. D. (2007). Printed graphene circuits. Adv. Mater., 19(21), 3623-3627.

Cheng, F., Liang, J., Tao, Z., \& Chen, J. (2011). Functional materials for rechargeable batteries. Adv. Mater., 23, 1695-1715.

Choucair, L. M., Thordarson, P., \& Stride, J. A. (2009). Gram-scale production of graphene based on solvothermal synthesis and sonication. Nature Nanotech., 4, 30.

Choucair, M., Thordarson, P., \& Stride, J. A. (2009). Gram-scale production of graphene based on solvothermal synthesis and sonication. Nature Nanotech., 4, 30.

Compton, O. C., Jain, B., Dikin, D. A., Abouimrane, A., Amine, K., \& Nguyen, S. T. (2011). ACS Nano, $5,4380$.

Cooper, D. R., Anjou, B. D., Ghattamaneni, N., Harack, B., Hilke, M., Horth, A., Majlis, N., Massicotte, M., Vandsburger, L., Whiteway, E., \& Yu, V. (2012). Experimental review of graphene. arXiv:1110.6557.

Coraux, J., Diaye, A. T., Busse, C., \& Michely, T. (2008). Nano Lett., 8, 565.

Craciun, M. F., Russo, S., Yamamoto, M., \& Tarucha, S. (2011). Tuneable electronic properties in graphene. Nano Today, 6(1), 42-60. ISSN 1748-0132.

Cricchio, D., Corso, P. P., Fiordilino, E., Orlando, G., \& Persico, F. (2009). A paradigm of fullerene. Journal Physics B., 42(8), 1-7, 085404.

Cullity, B. D., \& Stock, S. R. (2001). Elements Of X Ray Diffraction (3rd ed.). Upper Saddle River, NJ: Prentice Hall, New Jersey.

De, S., \& Coleman, J. N. (2010). Are there fundamental limitations on the sheet resistance.

De, S., Lyons, P. E., Doherty, E. M., Blau, W., Coleman, J., Nirmalraj, P. N., Boland, J. J., \& Higgins, T. (2009). Silver Nanowire Networks as Flexible, Transparent, Conducting Films: Extremely High DC to Optical Conductivity Ratios. ACS Nano, 3(7), 1767-1774.

Dean, C. R., Young, A. F., Meric, I., Lee, C., Wang, I., Sorgenfrei, S., Watanabe, K., Taniguchi, T., Kim, P., Shepard, K. L., \& Hone, J. (2010). Boron nitride substrates for high-quality graphene electronics. Nature Nanotech., 5, 722-726.

Di Vincenzo, D. P., \& Mele, E. J. (1984). Physics Review., B 29, 1685.

Dibenedetto, S. A., Facchetti, A., Ratner, M. A., \& Marks, T. (2009). Molecular self-assembled monolayers and multilayers for organic and unconventional inorganic thin-film transistor applications. Adv. Mater., 21, 1407-33.

Dikin, D. A., Stankovich, S., Zimney, E. J., Piner, R. D., Dommett, G. H. B., Evmenenko, G., Nguyen, S. T., \& Ruoff, R. S. (2007). Preparation and characterization of graphene oxide paper. Nature, 448, 457-460.

Dreyer, D. R., Park, S., Bielawski, C. W., \& Ruoff, R. S. (2010). Chem. Soc. Rev., 39, 228.

Earnshaw, A., \& Harrington, T. J. (1972). The chemistry of the transition elements. Oxford University Press.

Eda, G., Fanchini, G., \& Chhowalla, M. (2008) Large-area ultrathin films of reduced graphene oxide as a transparent and flexible electronic material. Nat. Nanotechnol., 3(5), 270-274.

Eizenberg, M., \& Blakely, J. M. (1979). Surf. Sci., 82, 228.

Elias, D. C., Nair,R. R., Mohiuddin, T. M. G., Morozov, S. V., Blake, P., Halsall, M. P., Ferrari, A. C., Boukhvalov, D. W., Katsnelson, M. I., Geim, A. K., \& Novoselov, K. S. (2009). Control of graphene'sproperties by Reversible Hydrogenation, Evedence for graphene. Science, 323, 610.

Emtsev, K. V., Bostwick, A., \& Horn, K. (2009). Towards wafer-size graphene layers by atmospheric pressure graphitization of silicon carbide. Nature Materials, 8, 203-207. 
Fan, L., Zou, J., Li, Z., Li, X., Wang, K., \& Wei, J. (2012). Topology evolution of graphene in chemical vapor deposition, a combined theoretical/experimental approach toward shape control of graphene domains, 115605.

Fan, X., Peng, W., Li, Y., Li, X., Wang, S., Zhang, G., \& Zhang, F. (2008). Adv. Mater., 20, 4490.

Feynman, R. (1959). There's Plenty of Room at the Bottom, Annual meeting of American Physical Society, California Institute of Technology.

Filleter, T., Emtsev, K. V., Seyller, T. \& Bennewitz, R. (2008). Local work function measurements of epitaxial graphene. Appl. Phys. Lett., 93, 133117.

Fisher, P. J., Srivastava, N., Feenstra, R. M., \& Sun, Y. (2009). Morphology of Graphene on SiC (0001) Surfaces.

Gaidau, C., Petica, A., Ignat, M., Iordache, O., \& Ditu, L. (2016). Enhanced photocatalysts based on Ag-TiO 2 and Ag-N-TiO 2 nanoparticles for multifunctional leather surface coating, 383-392.

Ge, W., Lu, B., Li, W., Lu, J., \& Ye, Z. (2013). Synthesis of graphene together with undesired CuxO nanodots on copper foils by low-pressure chemical vapor deposition (Vol. 97, pp. 9-14).

Geim, A. K., \& Novoselov, K. S. (2007). The rise of graphene. Nat. Mater., 6(3), 183-191.

Geng, H., Kim, K. K., So, K. P., Lee, Y. S., Chang, Y., \& Lee, Y. H. (2007). Effect of Acid Treatment on Carbon Nanotube-Based Flexible Transparent Conducting Films. J. Am. Chem. Soc., 1297758-7759.

Ghosh, S., Bao, W., Nika, D. L., Subrina, S., Pokatilov, E. P., Lau, C. N., \& Balandin, A. A.(2010). Dimensional crossover of thermal transport in few-layer graphene. Nat. Mater., 9, 555-558.

Giovannetti, G., Khomyakov, P. A., Brocks, G., Karpan, V. M., van den Brink, J., \& Kelly, P. J. (2008). Doping graphene with metal contacts. Phys. Lev. Lett., 101, 026803.

Golabi, S. M., \& Nozad, A. (2002). Electrocatalytic oxidation of methanol on electrodes modified by platinum microparticles dispersed into poly (o-phenylenediamine) film. J. Electroanal. Chem., 521, 161-167.

Granqvist, C. G. (2007). Transparent conductors as solar energy materials: apanoramic review. Sol. Energy Mater. Sol. Cells, 91, 1529-1598.

Gunes, F., Shin, H. J., Biswas, C., Han, G. H., \& Kim, E. S. (2010). Layer-by-layer doping of few-layer graphene film. ACS Nano, 4, 4595-600.

Guo, H. L., Wang, X. F., Qian, Q. Y., Wang, F. B., \& Xia, X. H. (2009). ACS Nano, 3, 2653.

Hamberg, I., \& Granqvist, C. G. (1986). Evaporated Sn-doped In2O3 films: Basic optical properties and applications to energy-efficient windows. J. Appl. Phys., 60, R123-R160.

Hamilton, J. C., \& Blakely, J. M. (1980). Surf. Sci., 91, 199.

Han, M., Ozyilmaz, Y., B., Zhang, Y., \& Kim, P. (2007). Phys. Rev. Lett., 98, 206805.

Han, T., Lee, Y., Choi, M., Woo, S., Bae, S., Hong, B. H., \& Lee, T. (2012). Extremely efficient flexible organic light-emitting diodes with modified graphene anode, 6(2), 105-110.

Hattab, H., Diaye, A. T., Wall, D., Klein, C., Jnawali, G., Coraux, J., Busse, C., Gastel, R. V., Poelsema, B., Michely, T., Meyer, F. J, Heringdorf, Z. U., \& Hoegen, H. M. (2012). Interplay of wrinkles, strain, and lattice parameter in graphene on iridium. Nano Lett., 12(2), 678-82.

Hernandez, Y., Nicolosi, V., Lotya, M., Blighe, F. M., Sun, Z., De, S., \& Andrea, C. (2008). High yield production of graphene by liquid phase exfoliation of graphite, 1-14.

Hibino, H., Kageshima, H., Kotsugi, M., Maeda, F., Guo, F. Z., \& Watanabe, Y. (2009). Dependence of electronic properties of epitaxial few-layer graphene on the number of layers investigated by photoelectron emission microscopy. Phys. Rev., B 79, 125437.

Hill, E. W., Vijayaragahvan, A., \& Novoselov, K. (2011). Graphene sensors. IEEE Sens. J., 11, 3161-3170.

Himpsel, F. J., Christmann, K., Heimann, P., Eastman, D. E., \& Feibelman, P. J. (1982). Surf. Sci., 115, L159.

Holland, L., \& Siddall, G. (1953). The properties of some reactively sputtered metal oxide films. Vacuum, 3, 375-391.

Hummers, W. S., \& Offeman, R. E. (1958). Journal of American. Chemical. Soceity., 80, 1339.

Hwang, J., Choi, H. K., Moon, J., Kim, T. Y., Shin, J., Hwang, J., \& Shin, J. (2012). Multilayered graphene anode for blue phosphorescent organic light emitting diodes. Appl. Phys. Lett., 100. 
Islam, M. R., \& Podder, J. (2009). Optical properties of $\mathrm{ZnO}$ nano fibre thin films grown by spray pyrolysis of Zinc Acetate Precursor. Crystal Research Technology, 44(3), 286-292.

Jafari, A., Ghoranneviss, M., \& Gholami, M. (2015). The role of deposition temperature and catalyst thickness in graphene domains on Cu. International Nano Letters, 5(4),199-204.

Janata, J., \& Josowicz, M. (2003).Conducting polymers in electronic chemical sensors. Nature Mater., 2, 19-24.

Jeffy, G. S. (2013). Electronic Theses and Dissertations UC Riverside Low Temperature Direct Growth of Graphene Films on Transparent Substrates by Chemical Vapor Deposition.

Jia, C., Jiang, J., Gan, L., \& Guo, X. (2012). Direct Optical Characterization of Graphene Growth and Domains on Growth Substrates, 1-6.

Jones, A. A., \& Safron, N. (2004). Mechanical Exfoliation to Make Graphene and Visualization Mechanical Exfoliation to Make Graphene and Visualization, 2-4.

Jun, S. C. (2015). Fundamental of Graphene, Graphene-based Energy Devices (1st ed.). Edited by ARashid bin Mohd Yusoff. Wiley-VCH Verlag GmbH \& Co. KGaA. Retrieved from http://www.nature.com/nnano 25/12/16 3:30pm

Kang, J., Kim, H., Kim, K. S., Lee, S.-K., Bae, S., Ahn, J.-H., Kim, Y.-J., Choi, J.-B., \& Hee Hong, B. H. (2011). High-performance graphene-based transparent flexible heaters. Nano Lett., 11, 5154-8.

Kedzierski, J., Hsu, P., Reina, A., Kong, J., Healey, Wyatt, P., \& Keast, C. (2009). Graphene-on-Insulator Transistors Made Using Con Ni Chemical-Vapor Deposition.

Kholin, A., Rut'kov, E. V., \& Tontegode, A. Y. (1985). Sov. Phys. Solid State, 27, 155.

Kim, K. S., Ozyilmaz, B., Ahn, J. H., Hong, B. H., \& Iijima, S. (2010). Enhancing the conductivity of transparent graphene films via doping. Nanotechnology, 5, 574.

Kim, K. S., Zhao, Y., Jang, H., Lee, S. Y., Kim, J. M., Kim, K. S., Ahn, J. H., Kim, P., Choi, J. Y., \& Hong, B. H. (2009). Large-scale pattern growth of graphene films for stretchable transparent electrodes. Nature, 457, 706-710.

Kim, K., Lee, Z., Malone, B. D., Chan, K. T., Aleman, B., Regan, W., Gannett, W., Crommie, M. F., Cohen, M. L., \& Zettl, A. (2011). Multiply folded graphene. Phys. Rev., B 83, 245433.

Kraus, J., Bocklein, S., Reichelt, R., Gunther, S., Santos, B., Mentes, T. O., \& Locatelli, A. (2013). Towards the perfect graphene membrane-improvement and limits during formation of highquality graphene grown on Cu-foils. Carbon, 64, 377-390.

Krishnamoorthy, K., Veerapandian, M., Yun, K., \& Kim, S. J. (2013). Carbon, 53, 38-49.

Kumar, A., Voevodin, A. A., Zemlyanov, D., Zakharov, D. N., \& Fisher, T. S. (2012). Rapid synthesis of few layer graphene over $\mathrm{Cu}$ foil. Carbon, 50, 1546-1553.

Lee, C., Wei, X., Kysar, J. W., \& Hone, J. (2008a). Measurement of the Elastic Properties and Intrinsic Strength of Monolayer Graphene. Science, 321(5887), 385-388

Lee, D. W., De, L., Santos, V. L., Seo., J. W., Felix, L. L., Bustamante, D. A., Cole, J. M., \& Barnes, C. H. W. (2010a). J. Phys. Chem., B 114, 5723-5728.

Lee, E. P., Peng, Z., Chen, W., Chen, S., Yang, H., \& Xia, Y. (2008c). ACS Nano, 2, 2167.

Lee, J. Y., Connor, S. T., Cui, Y., \& Peumans, P. (2008b). Solution-processed metal nanowire mesh transparent electrodes. Nano Lett., 8, 689-692.

Lee, W. H., Cho, J. H., \& Cho, K. (2010b). Control of mesoscale and nanoscale ordering of organic semiconductors at the gate dielectric semiconductor interface for organic transistors. J. Mater. Chem., 20, 2549-61.

Lee, Y., \& Ahn, J. (2013). Graphene based transparent conductivity films. NANO: Brief Rep. Rev., 8, 1-16, 1330001 .

Li, X. S., Cai, W. W., Colombo, L., \& Ruoff, R. S. (2009b). Evolution of Graphene Growth on Ni and Cu by Carbon Isotope Labeling. Nano Lett., 9, 4268-4272.

Li, X. S., Magnuson, C. W., \& Venugopal, A. (2011). Large-Area Graphene Single Crystals Grown by Low-Pressure Chemical Vapor Deposition of Methane on Copper. Journal of the American Chemical Society, 133(9), 2816-2819. 
Li, X., Wang, X., Zhang, L., Lee, S., \& Dai, H. (2008). Science, 319, 1229.

Li, X., Zhu, Y., Cai, W., Borysiak, M., Han, B., Chen, D., Piner, R. D., Colombo, L., \& Ruoff, R. S. (2009). Transfer of large-area graphene films for high-performance transparent conductive electrodes. Nano Lett., 9 , 4359-63.

Li, Z., Wu, P., Wang, C., Fan, X., Zhang, W., Zhai, X., Zeng, C., Li, Z., Yang, J., \& Hou, J. (2011). Low-temperature growth of graphene by chemical vapor deposition using solid and liquid carbon sources. American Chemical Society Nano, 5(4), 3385-3390.

Lianga, M., \& Zhi, L. (2009). Graphene-based electrode materials for rechargeable lithium batteries. J. Mater. Chem., 19(33), 5871-5878.

Lin, Y. M., Dimitrakopoulos, C., Jenkins, K. A., Farmer, D. B., Chiu, H. Y., Grill, A., \& Avouris, P. (2010). $100-\mathrm{GHz}$ transistors from wafer-scale epitaxial graphene. Science, 327(5966), 662-662.

Lin, Y. M., Garcia, A. V., Han, Farmer, D. B., Meric, Y. S., Wu, Y., Dimitrakopoulos, C., Jenkins, K. A., Farmer, D. B., Chiu, H. Y., Grill, A., Avouris, Ph., \& Jenkins, K. A. (2011). Wafer-scale graphene integrated circuit. Science, 332(6035), 1294-1297.

Liu, L., Ryu, S., Tomasik, M. R., \& Stolyarova, E. (2008). Graphene oxidation: Thickness-dependent etching and strong chemical doping. Nano Lett., 8, 1965-1970.

Liu, M., Yin, X., Ulin-Avila, E., Geng, B., Zentgraf, T., Ju, L., Wang, F., \& Zhang, X. (2011). A graphene-based broadband optical modulator. Nature, 474(7349), 64-67.

Loginova, E., Bartelt, N. C., Feibelman, P. J., \& McCarty, K. F. (2008). Evidence for graphene growth by Ccluster attachment. New Journal Physics, 10, 093026.

Losurdo, M., Giangregorio, M. M., Capezzuto, P., \& Bruno, G. (2011). Graphene CVD growth on copper and nickel: Role of hydrogen in kinetics and structure. Physical Chemistry Chemical Physics, 13(46), 20836-20843.

Lu, X. K., Yu, M. F., Huang, H., \& Ruoff, R. S. (1999). Nanotechnology, 10, 269.

Luo, Z., Lu, Y., Singer, D. W., Berck, M. E., Somers, L. A., Goldsmith, B. R., \& Charie Johnson, A. T. (2011). Effect of substrate roughness and feedstock concentration on growth of wafer-scale graphene at atmospheric pressure. Chem. Mater, 23, 1441-1447.

Mark, F., Goerbig, O., \& Notes, L. (2008). Introduction to the Physical Properties of Graphene.

McCleverty, J. (1999). Chemistry of the first-row Transition Metals. Oxford science publications.

McClure, J. W. (1956). Physical Review, 104, 666.

Meyer, J. C., Geim, A. K., Katsnelson, M. I., Novoselov, K. S., Booth, T. J., \& Roth, S. (2007). The structure of suspended graphene sheets. Nature, 446, 60-63.

Miao, C., Zheng, C., Liang, O., \& Xie, Y. (2011). World's largest Science, Technology and Medicine Open Access book publisher Chemical Vapor Deposition of Graphene. Retrieved from http://cdn.intechopen.com/pdfs-wm/15268.pdf

Minami, T. (2005). Transparent conducting oxide semiconductors for transparent electrodes. Semicond. Sci. Technol., 20, 535-544

Moed, H. F. (2000). Bibliometric Indicators Reflect Publication and Management Strategies Scientometrics, 47(2), 323-346.

Mohanty, N., \& Berry, N. (2008). Nano Lett., 8, 4469.

Mohanty, N., Moore, D., Xu, Z., Sreeprasad, T. S., Nagaraja, A., Rodriguez, A. A., \& Berry, V. (2012). Nanotomy based production of transferrable and dispersible graphene nanostructures of controlled shape and size. Nat. Commun., 3(5), 844-848.

Morgan, A. E., \& Somorjai, G. A. (1968). Low Energy Electron Diffraction Studies of Gas Adsorption on Platinum (100) Single Crystal Surface. Surf Sci., 12(3), 405.

Naghdi, S., Rhee, K. Y., Kim, M. T., Jaleh, B., \& Park, S. J. (2016). Atmospheric chemical vapor deposition of graphene on molybdenum foil at different growth temperatures. Carbon Letters, 18, 37-42.

Nair, R. R., Blake, P., Grigorenko, A. N., Novoselov, K. S., Booth, T. J., Stauber,T., Peres, N. M. R., \& Geim, A. K. (2008). Fine structure constant defines visual transparency of graphene. Science, 320(5881), 1308. 
Ni, Z. H., Wang, H. M., Kasim, J., Fan, H. M., Yu, T., Wu, Y. H., Feng, Y. P., \& Shen, Z. X. (2007). Nano Letter., 7, 2758.

Ni, Z., Wang, Y., Yu, T., \& Shen, Z. (2008). Raman Spectroscopy and Imaging of Graphene. Nano Res, 1, 273-291.

Novoselov, K. S., Falko, V. I., Colombo, L., Gellert, P. R., Schwab, M. G., \& Kim, K. (2012). Nature, 490, 192.

Novoselov, K. S., Geim, A. K., Morozov, S. V., Jiang, D., Zhang, Y., Dubonos, S. V., Grigorieva, I. V., \& Firsov, A. A. (2004). Electric field effect in atomically thin carbon films. Science, 306, 666-669.

Ong, M. T., \& Reed, E. J. (2012). Engineered piezoelectricity in graphene. ACS Nano, 6(2), 1387-1394.

Oshima, C., \& Nagashima, A. (1997). Ultra-thin epitaxial films of graphite and hexagonal boron nitride on solid surfaces. J. Phys.: Condens. Matter, 9, 1-20

Page, Z. A., Liu, Y., Duzhko, V. V., Russell, T. P., \& Emrick, T.(2014). Fulleropyrrolidine interlayers: Tailoring electrodes to raise organic solar cell efficiency. Science, 346, 441-444.

Panchal, V., Pearce, R., Yakimova, R., Tzalenchuk, A., \& Kazakova, O. (2013). Standardization of surface potential measurements of graphene domains. Sci. Rep., 3.

Pham, V. H., Cuong, T. V., Nguyen-Phan, T. D., Pham, H. D., Kim, E. J., Hur, S. H., Shin, E. W., Kim, S., \& Chung, J. S. (2010). Chem. Commun., 46, 4375.

Ponomarenko, L. A., Schedin, F., Katsnelson, M. I., Yang, R., Hill, E. W., Novoselov, K. S., \& Geim, A. K. (2008). Chaotic Dirac billiard in graphene quantum dots. Science, 320(5874), 356-358.

Qu, J., Shen, Y., Qu, X., \& Dong, S. (2004). Preparation of hybrid thin film modified carbon nanotubes on glassy carbon electrode and its electrocatalysis for oxygen reduction. Chem. Commun., 1, 34-35.

Rao, C. N. R., Sood, A. K., Subrahmanyam, K. S., \& Govindaraj, A. (2009). Angew. Chem. Int. Ed., 48, 7752.

Rao, C. N. R., Subrahmanyam, K. S., Ramakrishna-Matte, H. S. S., Abdulhakeem, B., Govindaraj, A., Das, B., Kumar, P., Ghosh, A., \& Late, D. J. (2010). Sci. Technol. Adv. Mater., 11, 054502.

Rao, C. N., \& Sood, A. K. (2013). Graphene Synthesis, Properties, and Phenomena (1st ed.). Wiley-VCH Verlag.

Reich, S., Maultzsch, J., Thomsen, C., \& Ordejón, P. (2002). Tight-binding description of graphene. Phys. Rev. B, 66(3), 035412.

Riaz, M., Fulati, A., Amin, G., Alvi, N. H., Nur, O., \& Willander, M. (2009). Journal of Applied Physics, 106, 034309.

Riaz, M., Fulati, A., Zhao, Q. X., Nur, O.,Willander, M., \& Klason, P. (2008). Nanotechnology, 19, 415708.

Royal, T. H., Academy, S. E., \& Sciences, O. F. (2010). Compiled by the Class for Physics of the Royal Swedish Academy of Sciences Graphene, 50005, 0-10.

Sahu, D. R., Lin, S., \& Huang, J. (2006). ZnO / Ag / ZnO multilayer films for the application of a very low resistance transparent electrode, 252, 7509-7514.

Sahu, S. R., Devi, M. M., Mukherjee, P., Sen, P., \& Biswas, K. (2013). Optical Property Characterization of Novel Graphene- $X(X=A g, A u$ and $\mathrm{Cu}$ ) Nanoparticle Hybrids. Journal of Nanomaterials, 232(409), 9.

Sarno, M., Cirillo, C., Piscitelli, R., \& Ciambelli, P. (2013). A study of the key parameters, including the crucial role of $\mathrm{H} 2$ for uniform graphene growth on Ni foil. J. Mol. Catal. A Chem., 366, 303-314.

Schedin, F., Geim, A. K., Morozov, S. V., Hill, E. W., Blake, P., Katsnelson, M. I., \& Novoselov, K. S. (2007). Nat. Mater., 6, 652.

Schwierz, F. (2010). Graphene transistors. Nat Nano, 5, 487-496.

Semenoff, G. W. (1984). Physical Review Letters, 53, 2449.

Sheraw, C. D., Zhou, L., Huang, J. R., Gundlach, D. J., \& Jackson, T. N. (2002). Organic thin-fi $\operatorname{lm}$ transistor-driven polymer dispersed liquid crystal displays on flexible polymeric substrates. Appl. Phys. Lett., 80, 1088-1090.

Shin, H. J., Kim, K. K., Benayad, A., Yoon, S. M., Park, H. K., Jung, I. S., Jin, M. H., Jeong, H. K., Kim, J. M., Choi, J. Y., \& Lee, Y. H. (1987). Adv. Funct. Mater., 19.

Singh, V., Joung, D., Zhai, L., \& Das, S. (2011). Progress in Materials Science Graphene based materials: Past, present and future. Progress in Materials Science, 56(8), 1178-1271. 
Stallings, J., Vance, E., Yang, J., Vannier, M. W., Liang, J., Pang, L., Liang Dai, Ye, I., \& Wang, G. (2013). Determining scientific impact using a collaboration index. Proceedings of the National Academy of Sciences.

Stankovich, S., Dikin, D. A., Piner, R. D., Kohlhaas, K. A., Kleinhammes, A., Jia, Y., Wu, Y., Nguyen, S. T., \& Ruoff, R. S. (2007). Carbon, 45, 1558.

Stankovich, S., Dikin, D. A., Piner, R. D., Kohlhaas, K. A., Kleinhammes, A., Jia, Y., \& Wu, Y. (2007). Synthesis of graphene-based nanosheets via chemical reduction of exfoliated graphite oxide, 45, 1558-1565.

Stöberl, U., Wurstbauer, U., Wegscheider, W., Weiss, D., Eroms, J., Stöberl, U., \& Eroms, J. (2008). Morphology and flexibility of graphene and few-layer graphene on various substrates Advertisement, 51906, 98-101.

Subrahmanyam, K. S., Panchakarla, L. S., Govindaraj, A., \& Rao, C. N. R. (2009). J. Phys. Chem.C, 113, 4257.

Sun, T., Wang, Z. L., Shi, Z. J., Ran, G. Z., Xu, W. J., Wang, Z. Y., Li, Y. Z., Dai, L., \& Qin, G. G. (2010). Multilayered graphene used as anode of organic light emitting devices. Appl. Phys. Lett., 96, 13301-13303.

Sundaram, R. S., Gomez-Navarro, C., Balasubramanian, K., Burghard, M., \& Kern, K. (2008). Adv. Mater., 20 , 3050 .

Sutter, P. (2009). Epitaxial graphene: How silicon leaves the scene. Nature Materials, 8(3), 171-2.

Sutter, P. W., Flege, J. I., \& Sutter, E. A. (2008). Epitaxial graphene on ruthenium, 7, 406.

Taghioskoui, M. (2009). Trends in graphene. Materials Today, 12(10), 34-37.

Tao, Z., Sheng-ke, Y., Min, Z., Yue, Z. C., \& Jing, C. (2011).The research of preparation and catalytic property of the titanium plate which loaded with the graphene modified $\mathrm{SnO}_{2}$. Water Resour. Environ. Prot. (ISWREP) Int. Symp., 2, 1501-1503.

Terasawa, T., \& Saiki, K. (2012). Growth of graphene on $\mathrm{Cu}$ by plasma enhanced chemical vapor deposition. Carbon, 50, 869-874.

Teweldebrhan, D., \& Balandin, A. A. (2009). Modification of graphene properties due to electron-beam irradiation. Appl. Phys. Lett., 94, 013101.

Thomas Reuters Web of science. (2013). Retrieved from http://isiknowledge.com

Tian, N., Zhou, Z. Y., Sun, S. G., Ding, Y., \& Zhong, L. W. (2007). Synthesis of tetrahexahedral platinum nanocrystals with high index facets and high electro-oxidation activity. Science, 316(5825), 732-735.

Traversi, F., Russo, V., \& Sordan, R. (2009). Integrated complementary Integrated complementary graphene inverter. Appl. Phys. Lett., 94(22), 1-3, 223312.

Unarunotai, S., Murata, Y., Chialvo, C. E., Kim, H. S., MacLaren, S., Mason, N., Petrov, I., \& Rogers, J. A. (2009). Appl. Phys. Lett., 95, 202101.

Vander-Pauw, L. J. (1958). A method of measuring specific resistivity and Hall Effect of discs. of arbitrary shape. Philips Res. Rep, 13(1).

Vlassiouk, I., Fulvio, P., Meyer, H., Lavrik, N., Dai, S., Datskos, P., \& Smirnov, S. (2013). Large scale atmospheric pressure chemical vapor deposition of graphene.

Vlassiouk, I., Regmi, M., Fulvio, P., Dai, S., Datskos, P., Eres, P., \& Smirnov, S. (2011). Role of Hydrogen in Chemical Vapor Deposition Growth of Large Single-Crystal Graphene. ACS Nano, 5(7), 6069-6076.

Wallace, P. R. (1947). Physical Review, 71, 476.

Wang, F., Zhang, Y. B., Tian, C. S., Girit, C., Zettl, A., Crommie, M., \& Shen, Y. R. (2008). Gate variable optical transitions in graphene. Science, 320, 206-209.

Wang, H., Nezich, D., Kong, J., \& Palacios, T. (2009). Graphene frequency multipliers. IEEE Electron Device Lett., 30(5), 547-549.

Wang, T. H., Yang, J.-C., \& Hsiao, Y.-J. (2011). Mechanical characterization of nanoindented graphene via molecular dynamics simulations. Nanoscale Research Letters, 20116, 481.

Wang, X., Li, X., Zhang, L., Yoon, Y., Weber, P. K., \& Wang, H. (2009). N-doping of graphene through electrothermal reactions with ammonia. Science, 324(5928), 768-771.

Wang, Y., Shi, Z., Huang, Y., Ma, Y., Wang, C., \& Chen, M. (2009). Supercapacitor devices based on graphene materials. J. Phys. Chem. C., 113(30), 13103-13107. 470-473.

Wassei, J. K., \& Kaner, R. B. (2010). Mater. Today, 13, 52. 
Wassei, J. K., Mecklenburg, M. I., Torres, J. A., Fowler, J. D., Regan, B. C., Kaner, R. B., \& Weiller, B. H. (2012). Chemical vapor deposition of graphene on copper from methane, ethane and propane: Evidence for bilayer selectivity, 8(9), 1415-1422.

Wu, B., Tuncer, H. M., Naeem, M., Yang, B., Cole, M. T., William, I., Milne, W. I., \& Hao, Y. (2014). Experimental demonstration of a transparent graphene millimetre wave absorber with $28 \%$ fractional bandwidth at $140 \mathrm{GHz}$. Sci. Rep., 4, 1-7, 4130.

Wu, J., Wang, Y., Zhang, D., \& Hou, B. (2011). Studies on the electrochemical reduction of oxygen catalyzed by reduced graphene sheets in neutral media. J. Power Sources, 196, 1141-1144.

Wu, M. S., \& Zheng, Y. J. (2013). Electrophoresis of randomly and vertically embedded graphene nanosheets in activated carbon film as a counter electrode for dye-sensitized solar cells. Phys. Chem. Chem. Phys., 15, 1782-1787.

Wu, Q., Xu, Y., Liu, A., \& Shi, G. (2010). Supercapacitors based on flexible graphene/polyaniline nanofiber composite films. ACS Nano, 4(4), 1963-1970.

Wu, S., Huangm, F., Lan, X., Wang, X., Wang, J., \& Meng, C. (2013). Electrochemically reduced graphene oxide and Nafion nanocomposite for ultralow potential detection of organophosphate pesticide. Sens Actuators B, $177,724-729$.

Wu, S., Lan, X., Cui, L., Zhang, L., Tao, S., Wang, H., Han, M., Liu, Z., \& Meng, C. (2011). Application of graphene for preconcentration and highly sensitive stripping voltammetric analysis of organophosphate pesticide. Anal Chim Acta, 699, 170-176.

Wu, X., Hu, Y., Ruan, M., Madiomanana, N. K., Berger, C., \& Heer, W. A. (2009). Thermoelectric effect in high mobility single layer epitaxial graphene. Appl. Phys. Lett., 95, 223108.

Wu, Y., Ruan, X., Chen, C., Shin, Y., Lee, Y., Niu, J., Liu, J., Chen, Y., Yang, K., Zhang,X. H., Ahn, J., \& Yang, H. (2013). Graphene/liquid crystal based terahertz phase shifters. Opt. Express, 21(1-8), 021395.

Wu, Z., Chen, Z., Du, X., Logan, J. M., Sippel, J., Nikolou, M., \& Rinzler, A. G. (2004). Transparent Conductive Carbon Nanotube Films. Science, 305(8), 1273-1276.

Xiao, D., Liu, G. B., Feng, W., Xu, X., \& Yao, W. (2012). Phys. Rev. Lett., 108, 196802.

Xiao, N., Dong, X., Song, L., Liu, D., Tay, Y., Wu, S., \& Zhao, Y. (2011). Enhanced Thermopower of Graphene Films with Oxygen Plasma Treatment. ACS Nano, 5(4), 2749-2755.

Xin, Y., Lui, J., Lui, W., Gao, J., Xie, Y., \& Yin, Y. (2011). Preparation and characterization of Pt supported on graphene with enhanced electrocatalytic activity in fuel cell. J. Power Sources, 196(3), 1012-1018.

Xue, J., Sanchez-Yamagishi, J., Bulmash, D., Jacquod, P., Deshpande, A., Watanabe, K., Taniguchi, T., Jarillo-Herrero, P., \& LeRoy, B. L. (2011). Scanning tunneling microscopy and spectroscopy of ultra-flat graphene on hexagonal boron nitride. Nature Mater, 10, 282-285.

Yan, Z., \& Barron, A. (2010). Characterization of Graphene by Raman Spectroscopy. Connexions, (6), 29.

Yang, H., Heo, J., Park, S., Song, H. J., Seo, D. H., Byun, K. E., Kim, P., Yoo, I., Chung, H. J., \& Kim, K. (2012). Graphene Barristor, a Triode Device with a Gate-Controlled Schottky Barrier. Science, 336, 1140-1143.

Yang, W., Gao, Z., Wang, J., Ma, J., Zhang, M., \& Liu, L. (2013). Solvothermal one-step synthesis of Ni-Al layerd doubled hydroxide/carbon nanotube/reduced graphene oxide sheet ternary nanocomposite with ultrahigh capacitance for supercapacitor. ACS Apply Material Interfaces, 5, 5443.

Yankowitz, M., Xue, J., Cormode, D., Sanchez-Yamagishi, J. D., Watanabe, K., Taniguchi, T., Jarillo-Herrero, P., Jacquod, P., \& LeRoy, B. J. (2012). Emergence of superlattice Dirac points in graphene on hexagonal boron nitride. Nature Phys., 8, 382-386.

Yu, Y. J., Zhao, Y., Ryu, S., Brus, L. E., Kim, K. S., \& Kim, P. (2009). Tuning the Graphene Work Function by Electric Field Effect. Nano Lett., 9, 3430-3434.

Yue, G., Lin, J., Taib, S., Xiaoa, Y., \& Wua, J. (2012). A catalytic composite film of $\mathrm{MoS}_{2} /$ graphene flake as a counter electrode for Pt-free dye-sensitized solar cells. Electrochim. Acta, 85, 162-168.

Zhang, J., Hu, P., Wang, X., \& Wang, Z. (2012). Structural evolution and growth mechanism of graphene domains on copper foil by ambient pressure chemical vapor deposition. Chem. Physics Letter, 536, 123-128.

Zhang, J., Yang, H., Shen, G., Cheng, P., Zhang, J., \& Guo, S. (2010). Chem. Commun., 46, 1112. 
Zhang, W. M., Wu, X. L., Hu, J. S., Guo, Y. G., \& Wan, L. J., (2008). Carbon coated Fe3O4 nanospindles as a superior anode material for lithium-ion batteries. Adv. Funct. Mater., 18, 3941-3946.

Zhou, M., Wang, Y., Zhai, Y., Zhai, J., Ren, W., Wang, F., \& Dong, S. (2009). Chem. Eur. J., 15, 6116.

Zhou, W., Apkarian, R., Wang, Z. L., \& Joy, D. (2007). Fundamentals of Scanning Electron Microscopy (SEM)Scanning Microscopy for Nanotechnology. Springer New York.

Zhou, X., Zhang, J., Wu, H., Yang, H., Zhang, J., \& Guo, S. (2011). J. Phys. Chem.C, 115, 11957.

Zhou, Y., Fox, D. S., Maguire, P., Connell, R. O., Masters, R., Rodenburg, C., \& Zhang, H. (2016). Quantitative secondary electron imaging for work function extraction at atomic level and layer identification of graphene. Nature Publishing Group, 4-11.

Zhu, C., Guo, S., Fang, Y., \& Dong, S. (2010). Reducing sugar: new functional molecules for the green synthesis of graphene nanosheets. ACS Nano, 4, 2429.

Zhu, Y., Murali, S., Cai, W., Li, X., Suk, J. W., Potts, J. R., \& Ruoff, R. S. (2010). Graphene and Graphene Oxide Synthesis, Properties, and Applications. Advanced Materials, 22, 3906-3924.

Ziegler, D., Gava, P., Guttinger, J., Molitor, F., Wirtz, L., Lzzeri, M., Saitta, A. M., Stemmer, A., Mauri, F., \& Stampfer, C. (2011). Variations in the work function of doped single- and few-layer graphene assessed by Kelvin probe force microscopy and density functional theory. Phys. Rev. B, 83, 235434.

\section{Copyrights}

Copyright for this article is retained by the author(s), with first publication rights granted to the journal.

This is an open-access article distributed under the terms and conditions of the Creative Commons Attribution license (http://creativecommons.org/licenses/by/4.0/). 